\title{
Identification of the Expression and Clinical Significance of E2F Family in Clear Cell Renal Cell Carcinoma
}

\author{
Ru Chen ${ }^{1,2, *}$, Zhicheng Zhang ${ }^{1, *}$, Bing $\mathrm{Hu}^{1, *}$, Ming Jiang ${ }^{1, *}$, Ping Zheng ${ }^{3}$, Wen Deng ${ }^{1}$, Bin Fu ${ }^{1,4}$, \\ Ting Sun ${ }^{1,4}$ \\ 'Department of Urology, The First Affiliated Hospital of Nanchang University, Nanchang City, 330000, Jiangxi Province, People's Republic of China; \\ ${ }^{2}$ Department of Urology, The First Hospital of Putian City, Putian, 35000I, Fujian, People's Republic of China; ${ }^{3}$ Department of Urology, Shangrao \\ municipal Hospital, Shangrao, 334000, Jiangxi Province, People's Republic of China; ${ }^{4}$ Jiangxi Institute of Urology, Nanchang City, 330000 , Jiangxi \\ Province, People's Republic of China
}

*These authors contributed equally to this work

Correspondence: Bin Fu; Ting Sun, Email urofbin@I63.com; 361439919033@email.ncu.edu.cn

Background: Multiple studies have identified that E2F transcriptions act as important regulators for the tumorigenesis and progression of several human cancers. However, little is known about the function of E2Fs in clear cell renal cell carcinoma (ccRCC).

Methods: We firstly investigated the expression levels, genetic alteration, and biological function of E2Fs in patients with ccRCC and the connections between the immune cell infiltration and the overall survivals of ccRCC patients with the E2Fs expression levels based on UALCAN, The Cancer Genome Atlas database, Gene Expression Profiling Interactive Analysis, TIMER, STRING, GSCALite and cBioPortal databases.

Results: Results revealed that the expression levels of E2F1/2/3/4/6/7/8 were markedly upregulated in patients with ccRCC, while the expression of E2F5 displayed an opposite trend. We also experimentally validated the overexpression of E2F3/4/7 in human ccRCC tissues and ccRCC cell lines. Furthermore, the high E2F1/2/3/4/7/8 expression levels were clearly associated with worse pathological characteristics of ccRCC, including high pathological stage, poor molecular subtypes and high tumor grade. Meanwhile, high expression levels of E2F1/2/4/7/8 were evidently associated with worse overall survivals (OSs) and progression-free survivals (PFSs) of patients harboring ccRCC. Univariate and multivariate analyses illustrated that the expressions of E2F4/5/7 were independent factors associated with the OSs and PFSs of patients with ccRCC. Meanwhile, the mutations in E2Fs were also significantly related to poor OSs and PFSs of patients with ccRCC. Mechanically, the E2Fs genes synergistically promoted the progression of ccRCC by accelerating the cell cycle and inhibiting DNA damage response and apoptosis after performing the protein structure, functional enrichment, and PPI network analyses. In addition, E2Fs genes were also significantly associated with tumor immune cells infiltration and the drug sensitivity in ccRCC.

Conclusion: As a result, E2F4/7 were highly expressed in ccRCC and significantly associated with worse pathological characteristics of ccRCC, including high pathological stage, poor molecular subtypes and high tumor grade, tumor immune cell infiltration, and drug sensitivity, consequently translating into poor OSs and PFSs of patients with ccRCC. Our results indicated that E2F4/7 could be potential biomarkers and therapeutic targets of ccRCC patients.

Keywords: clear cell renal cell carcinoma, E2Fs, prognosis, UALCAN, GEPIA

\section{Introduction}

E2F transcription factors (E2Fs) are a group of genes that codify a family of transcription factors (TFs) which consist of eight family members in the mammalian cells, which play a critical role in regulating the expression of many genes required for cell cycle progression, DNA repair, and apoptosis. ${ }^{1,2}$ The members of this family can be divided into two groups based on their specific functions. E2F1, E2F2, and E2F3a are considered to be transcriptional activators, while 
E2F3b and E2F4 to 8 are considered to be transcriptional repressors. ${ }^{3}$ E2F proteins are located at the "bottom" of the growth factor signaling cascade and make essential contributions to the process of cell cycle, so the deregulation of the members of the E2F family is closely related to the occurrence of several human cancers, such as lung cancer, ${ }^{4}$ gastric cancer, ${ }^{5}$ bladder cancer, ${ }^{6}$ prostate cancer, ${ }^{7}$ ovarian cancer, ${ }^{7}$ and breast cancer. ${ }^{8}$

According to the global cancer statistics, renal cell carcinoma (RCC) was the 16th most commonly diagnosed cancer worldwide, with approximately 431,288 new cases. Furthermore, RCC had the 16th highest cancer-related mortality with 179,368 deaths, accounting for $1.8 \%$ of all cancer-related deaths in the whole world. ${ }^{9}$ The incidence of kidney cancer has increased in many countries over the past decade. ${ }^{10}$ The clear cell RCC (ccRCC) stands for the most common histological forms of kidney cancer. ${ }^{11}$ Localized renal tumors were best managed by partial nephrectomy or radical nephrectomy, irrespective of the surgical approach, while embolization, radiofrequency ablation, and cryoablation were provided as alternatives to surgery. For patients with metastatic RCC, systemic therapies, including immunotherapy, targeted therapies, radiotherapy, cytoreductive nephrectomy, were developed. ${ }^{12}$ Notably, although the five-year survival proportion of patients bearing RCC in clinical stage I reached up to $80-95 \%$, this rate dramatically dropped down to less than $10 \%{ }^{13}$ in patients with RCC in clinical stage IV, of whom the average overall survival length is only $10-15$ months, implying the great clinical significance of early diagnosis and treatment for patients with RCC. ${ }^{14}$ However, there are no clinical prognostic biomarkers for predicting prognosis and early diagnosis. A better understanding of the mechanism of ccRCC tumorigenesis and development is needed to help to develop and improve the treatment of patients diagnosed with advanced ccRCC. Our exploration of the underlying mechanisms of E2Fs in ccRCC will help to provide new clinical biomarkers and therapeutic targets for RCC.

Up to now, eight members (E2F1-E2F8) have been identified in human genomes. E2Fs are thought to play unique and complex roles in kidney cancer. Many studies have reported that E2F1 has a role in carcinogenesis of ccRCC and is found to be significantly upregulated in cancer. Cen et $\mathrm{al}^{15}$ reported that the circSDHC competitively bound to miR-127-3p, preventing its inhibition of the downstream gene E2F1 pathway, leading to malignant progression of ccRCC. Knockdown of E2F1 in ccRCC can promote the expression levels of miR-520c-3p, miR-372-3p, and miR-373-3p and thereby inhibit SPOP signaling expression, which promotes cell proliferation. ${ }^{16}$ The aberrant expression of E2F1 plays a key role in driving human ccRCC invasion and metastasis. ${ }^{17}$ E2F1 activation can promote the proliferation, migration and invasion of cancer cells, so that renal clear cancer cells have higher malignancy and vascular infiltration potential. ${ }^{18}$ The lncRNA RCAT1 could protect E2F2 from mediating degradation by sponging miR-214-5p abundantly, which promoted cell proliferation, migration, and invasion. ${ }^{19}$ However, Gao et $\mathrm{al}^{20}$ reported that E2F2 acts as a tumor suppressor in ccRCC and participated in miR-155-regulated cancer cell proliferation and invasion. There are few studies on the role of E2F3 in kidney cancer. The miR-200c directly targets oncogene E2F3 and inhibits kidney cancer cell growth and metastasis. ${ }^{21}$ One study found that E2F4 ccRCC tumor cells showed higher nuclear expression of E2F4 than normal kidney samples and E2F4 might function as an oncogene during tumorigenesis of ccRCC. ${ }^{22}$ However, the role of E2F5 and E2F8 in kidney cancer has not been investigated. The up-regulation of E2F6 was found to promote cell-colony formation and to accelerate apoptosis by suppressing the inhibitory effect of miR-425. ${ }^{23}$ E2F7 was increased in patients with RCC and might serve as a potential target for RCC. ${ }^{24}$ To date, the distinct expression/mutation patterns and prognostic values of E2Fs in clear cell RCC have yet to be fully elucidated and remain unknown.

In the current study, we firstly explored the expression and mutation status of E2Fs in ccRCC with the multiple databases. In addition, the connections between the E2Fs expression levels with clinicopathological features, prognosis, tumor immune cell infiltration and the drug sensitivity in patients with ccRCC were also investigated. Furthermore, we mechanically interpreted these results by analyzing the protein structure, functional enrichment, and PPI network.

\section{Materials and Methods}

\section{Ethics Statement}

Our research protocol was approved by the Ethics Committee of the First Affiliated Hospital of Nanchang University. Data were retrieved from online databases, and tissue samples were collected from patients who had given permission for their samples to be used in research and admitted to the Department of Urology in hospital. 


\section{Patient and Tumor Samples}

Twenty paired KIRC (kidney renal clear cell carcinoma) and adjacent normal tissues were pathologically confirmed by two independent pathologists and subsequently included in this study. Twenty pairs of matched ccRCC tissues and adjacent normal kidney tissues were immediately frozen after resection and stored in liquid nitrogen until use in the First Affiliated Hospital of Nanchang University from 2019 to 2020. The extracted RNA was reversely transcribed into cDNA with the First-Strand cDNA Synthesis kit (Qiagen, USA) in accordance with the manufacturer's instructions. SYBR Real-Time PCR kit (Qiagen, USA) was employed to perform RT-qPCR under the specific conditions: $95^{\circ} \mathrm{C}$ for 2 min, followed by 40 cycles of $95^{\circ} \mathrm{C}$ for $5 \mathrm{~s}$ and $60^{\circ} \mathrm{C}$ for $10 \mathrm{~s}$. The $2-{ }^{\Delta \Delta \mathrm{Ct}}$ method was utilized to evaluate the relative gene expressions. Each analysis was performed in triplicate. In addition, $\beta$-actin was considered as an internal reference gene.

\section{Cell Lines and Cell Culture}

The KIRC cell lines (HK-2, 786-O, A498, OSRC-2, Caki-1, 769-P, ACHN), which were obtained from the ATCC, were cultured in MEM, RPMI-1640 and DMEM standard culture medium (GIBCO, Thermo Fisher Scientific, Inc.) supplemented with 10\% fresh fetal bovine serum before use (FBS; Gibco; Invitrogen; Thermo Fisher Scientific, Inc.).

\section{RNA and Quantitative Real-Time PCR}

Trizol reagent was used for total RNA isolation from the ccRCC tissues and adjacent non-tumor tissues and ccRCC cell lines. The $1 \mu \mathrm{g}$ RNA from each sample was used for cDNA synthesis (Tiangen) in accordance with the manufacturer's protocol. Primers used were listed as follows: GAPDH forward primer GCCACATCGCTCAGACACCAT, GAPDH reverse primer: CCCATACGACTGCAAAGACCC, E2F1 forward primer: TGCCACCATAGTGTCACCAC E2F1 reverse primer: GTTCTTGCTCCAGGCTGAGT, E2F2 forward primer: ACGCCCGTCTTAAAGTCCAG, E2F2 reverse primer: TAAATGGCAGCCCAGTGGAG, E2F3 forward primer: CCAGCCAACCTCTTACAGCA, E2F3 reverse primer: TCGAAGAGATCGCTGATGCC, E2F4 forward primer: TCACAGAGGACGTGCAGAAC, E2F4 reverse primer: CGGATGGCCAAGAGGGTATC, E2F5 forward primer: GGACCTATCCATGTGCTGCT, E2F5 reverse primer: GAGGAAGGCTGTGTGAGGTC, E2F6 forward primer: GGTGTGTTTCTGTGCACCTG, E2F6 reverse primer: ACCCACCAGTCCAGTGATGA, E2F7 forward primer: GTCGTCCACACTCCCTTCTG, E2F7 reverse primer: TGGATGTCCCACGTAAACGG, E2F8 forward primer: CGCCCAGTAGCCCTATCAAG, E2F8 reverse primer: CTGGTTTGCAGGGTCCAGAT. All qRT-PCR assays were completed with SYBR Real-Time PCR kit (Qiagen, USA) under the specific conditions: $95^{\circ} \mathrm{C}$ for $2 \mathrm{~min}$, followed by 40 cycles of $95^{\circ} \mathrm{C}$ for $5 \mathrm{~s}$ and $60^{\circ} \mathrm{C}$ for $10 \mathrm{~s}$. The $2-^{\Delta \Delta \mathrm{Ct}}$ method was utilized to evaluate the relative gene expressions. Each analysis was performed in triplicate. In addition, $\beta$-actin was considered as an internal reference gene.

\section{Statistical Analysis}

All statistical analyses were performed in R package (V3.6.2). The effect of clinical parameters and mRNA expression of E2Fs on survival along with other clinical characteristics (histologic grade and pathologic stage) of ccRCC patients was evaluated using univariate Cox regression. Then, those with $p$ value $\leq 0.1$ were retained for subsequent analysis. Furthermore, multivariate analysis using Cox regression was performed to verify the relationship between clinical characteristics and E2Fs expression. All data were analyzed with the Mann Whitney $U$-test for and $p$ value $<0.05$ was considered statistically significant.

\section{UALCAN}

The expression of E2Fs in ccRCC was determined based on the TCGA data using the UALCAN (http://ualcan.path.uab. edu/) database developed by Chandrashekar et al, ${ }^{25}$ which is an online bioinformatics tool for analyzing and visualizing TCGA. Correlation of the E2Fs expression with clinical pathological parameters was also analyzed by using the online bioinformatics tool. The analyses were performed based on the TCGA_KIRC data. A $p$-value $<0.05$ was considered significant $(p$-value $<0.05(*), p$-value $<0.01(* *), p$-value $<0.001(* * *)$ and $p$-value $<0.0001(* * * *))$. 


\section{GEPIA Dataset}

The GEPIA Dataset is an interactive web server for exploring large data sets, containing data from 9736 tumors and 8587 normal samples from The Cancer Genome Atlas and the Genotype Tissue Expression projects. ${ }^{26}$ In our research, we analyzed the expression levels of E2Fs genes in cancerous tissues and normal tissues and the prognostic value of candidate genes in TCGA using the GEPIA database. $P$ value $<0.05$ was considered significant.

\section{cBioPortal}

c-BioPortal (www.cbioportal.org) is an online open access resource that has been developed for comprehensive analysis of multiple cancer genomic datasets and clinical data from the TCGA database. ${ }^{27}$ In this study, we collected the genomic profiles corresponding to E2Fs family of tumor genomes, which contained mutations, putative copy-number alterations from GISTIC and mRNA expression z-scores (RNA Seq V2 RSEM). Furthermore, genetic mutations in E2Fs and their correlations with OS and DFS of KIRC patients were displayed online as Kaplan-Meier plots, and the Log rank test was performed to check the difference between altered group and unaltered group. Mutation rate means that genetic alteration in patients accounted for all the sequenced patients based on the cBioPortal database. ${ }^{27}$

\section{GSCALite}

GSCALite (http://bioinfo.life.hust.edu.cn/web/GSCALite/) is an important online website for the cancer genome analysis platform. Furthermore, we utilized it to analyze pathway activity, miRNA network and the drug sensitivity of the E2Fs family in ccRCC. The pathway activity module was used to analyze the relation between members of E2F family and pathway activity. The association between drug sensitivity and E2Fs genes expression profiling data of cancer cell lines was performed via the drug-sensitivity module.

\section{STRING Analysis}

A protein interaction network was constructed for differential genes using the online functional protein association networks database STRING (https://string-db.org/). ${ }^{28}$ In the study, the network for E2Fs and the 50 most frequently altered neighboring genes was constructed using the STRING online database.

\section{TIMER}

TIMER (http://cistrome.org/TIMER/) is a TCGA database visual website developed for analyses of tumor immunity. ${ }^{29}$ The correlation between the members of E2Fs family and the infiltration of immune cells in ccRCC was explored in the gene module. The somatic copy number alteration (SCNA) module of was used to associate genetic copy number alterations of E2Fs with the abundance of immune infiltrates.

\section{Results}

\section{Expression Levels of Different E2Fs in Patients with Clear Cell Renal Cell Carcinoma}

First, compared with paired normal tissue samples, the expression levels of E2Fs family in ccRCC samples were analyzed based on The Cancer Genome Atlas (TCGA) dataset (https://portal.gdc.cancer.gov/). As showcased in Figure 1A, the transcriptional levels of E2F1/2/3/4/6/7/8 ( $p<0.05$ for all) in ccRCC tissues were expressed at significantly higher levels compared with that in normal samples, while the E2F5 was significantly downregulated in renal cancer samples compared with normal samples. Next, we analyzed the eight E2Fs factors mRNA transcription levels of ccRCC patients according to the UALCAN database. As shown in Figure 1B-I, the transcriptional levels of E2F1, E2F2, E2F3, $\mathrm{E} 2 \mathrm{~F} 4, \mathrm{E} 2 \mathrm{~F} 6, \mathrm{E} 2 \mathrm{~F} 7$, and $\mathrm{E} 2 \mathrm{~F} 8$ in ccRCC tissues were significantly increased than in normal tissues while the transcriptional levels of E2F5/6 were decreased in normal tissues than in cancer tissues. Then, the mRNA expression levels of E2Fs members of the family were measured by RT-qPCR. We further verified the individual mRNA level of E2Fs in ccRCC tissues $(n=20)$ and normal kidney tissues $(n=20)$ by using cancer and adjacent normal tissues, which were obtained from patients. qRT-PCR was used to detect the mRNA levels of E2F1/2/3/4/5/6/7/8 in ccRCC tissues and paired-adjacent normal kidney tissues (Figure 2A-H, respectively). As a result, the transcriptional levels of E2F3/4/5/7 
A

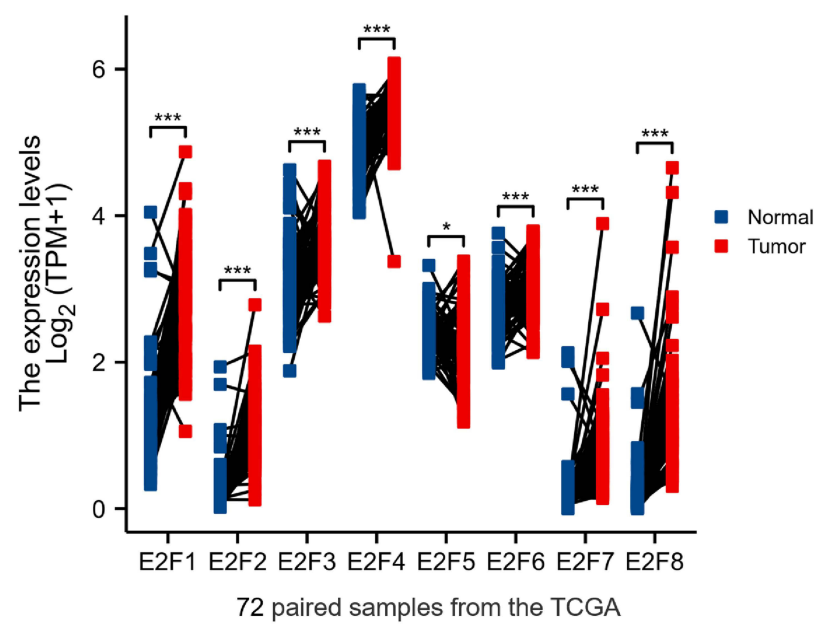

B

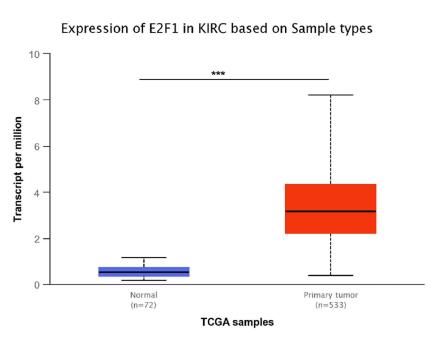

C

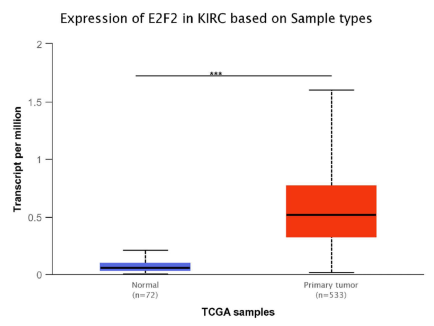

D

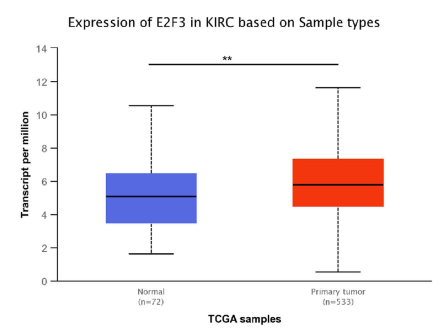

G

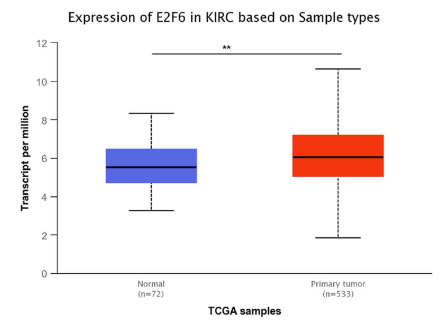

E

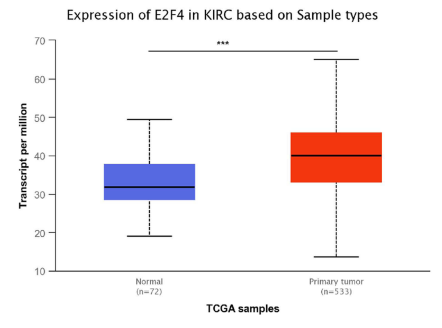

H

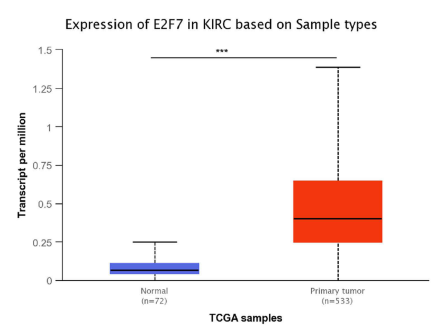

F

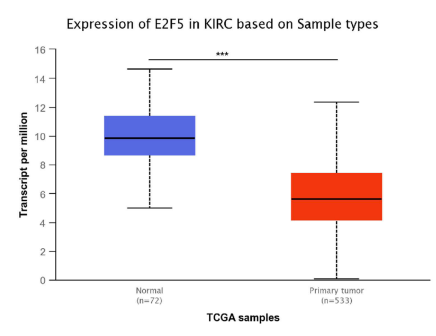

I

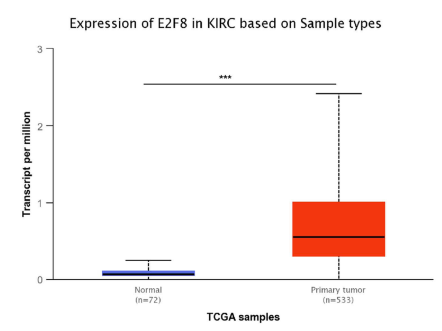

Figure I Transcriptional expression of E2Fs in 72 pairs of kidney cancer tissues and adjacent normal kidney tissues (TCGA and GTEx database) (A). mRNA expression of distinct E2Fs members in ccRCC tissues and normal kidney tissues (UALCAN) (B-I). ns indicates not significant; ${ }^{*} p<0.05$; $*_{p} p<0.01$; ***p $<0.00$ I. 

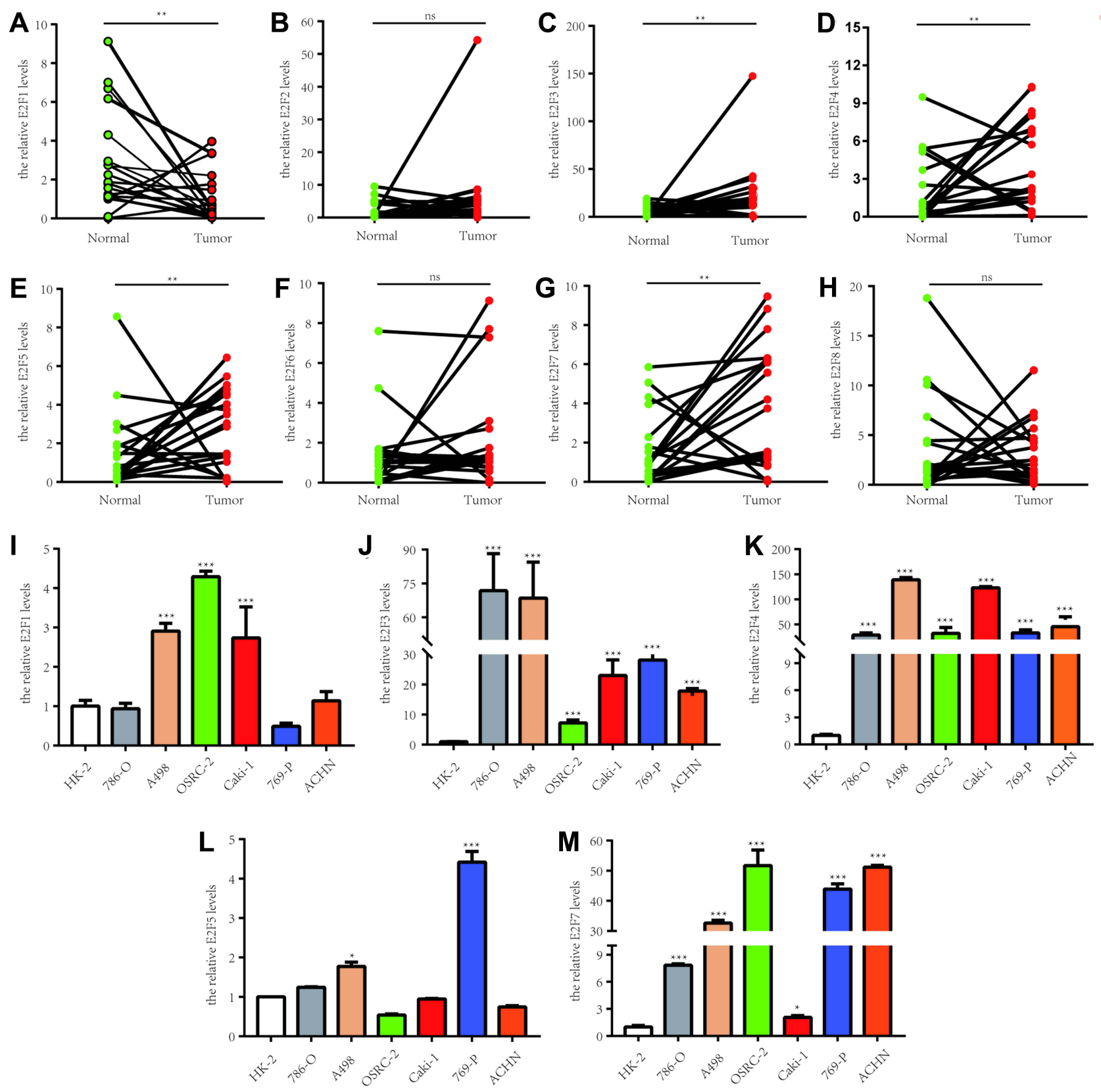

Figure 2 qRT-PCR were used to detect the mRNA levels of E2FI (A), E2F2 (B), E2F3 (C), E2F4 (D), E2F5 (E), E2F6 (F), E2F7 (G), E2F8 (H) in ccRCC tissues and pairedadjacent normal kidney tissues. Comparison of the mRNA levels of E2Fs in ccRCC cell lines between normal cell lines. The mRNA levels of E2FI (I), E2F3 (J), E2F4 (K), E2F5 $(\mathbf{L})$, and E2F7 (M) in kidney cancer and normal cell lines were estimated with qRT-PCR. ns indicates not significant; ${ }^{*} p<0.05 ; * * p<0.0$ I; *** $p<0.00$ I.

were decreased significantly in normal tissues relative to cancer tissues, while E2F1 was downregulated in the cancer tissues. The mRNA levels of E2F1/3/4/5/7 in kidney cancer and normal cell lines were estimated with qRT-PCR (Figure 2I-M, respectively). The results revealed that the transcriptional levels of E2F3/4/7 were upregulated in kidney cancer cell lines compared with normal cell lines.

\section{Association of mRNA Expression of Different E2Fs Family Members with Clinicopathological Parameters of ccRCC Patients}

After the relationship between mRNA expression of E2Fs was examined in ccRCC tissues and normal samples, then the relationship between the expression of different E2Fs mRNA and clinicopathological features was analyzed, including 
pathological stage, molecular subtypes and tumor grade. As presented in Figure 3A-H, the mRNA expressions of E2F1/ 2/7 were significantly related to patients' pathological stages, while mRNA expressions of E2F3/4/5/6/8 were not associated with patients' pathological stages. Patients with more advanced pathological stages tended to express higher mRNA expressions of E2F1/2/7 and the highest mRNA expressions of E2F1/2/7 were found in stage IV. The mRNA levels of E2F1/2/3/4/5/6/7/8 in good risk (ccA) and poor risk (ccB) subtypes of KIRC are shown in Figure 4A-H. the mRNA expression levels of E2F1/2/7/8 in the ccA subtype of ccRCC were significantly lower compared to the $\mathrm{ccB}$ subtype of ccRCC, while the expression of E2F6 produced the opposite result. Associations of E2F1/2/3/4/5/6/7/8 expressions with tumor grades of ccRCC are presented in Figure 5A-H, respectively. Similarly, mRNA expressions of E2F1/2/3/7/8 were remarkably associated with tumor grade and their mRNA expression levels of them increased as tumor grade increased. The highest mRNA expression of E2F4 was found in grade 1, while the highest mRNA expression of E2F5/6 was found in grade 2. Stated thus, the above results suggested that there be significant association between mRNA expressions of E2Fs and clinicopathological parameters.

\section{Prognostic Value of mRNA Expression of E2Fs in Patients with ccRCC}

We extracted the E2Fs level data and prognostic from TCGA to further detect the prognostic values of the mRNA expression of E2Fs in ccRCC patients. As shown in Figure 6A, most mRNA expressions of E2Fs family members were significantly correlated with ccRCC patients' prognosis. Our results showed that higher mRNA expression in $\mathrm{E} 2 \mathrm{~F} 1(\mathrm{HR}=1.67,95 \% \mathrm{CI}: 1.23-2.26$, and $p=0.001), \mathrm{E} 2 \mathrm{~F} 2(\mathrm{HR}=1.67,95 \% \mathrm{CI}: 1.23-2.27$, and $p=0.001)$, E2F3 $(\mathrm{HR}=1.67,95 \% \mathrm{CI}: 1.23-2.26$, and $p=0.001), \mathrm{E} 2 \mathrm{~F} 4(\mathrm{HR}=1.84,95 \% \mathrm{CI}: 1.36-2.50$, and $p<0.001), \mathrm{E} 2 \mathrm{~F} 5(\mathrm{HR}=1.66$, 95\% CI:1.23-2.26, and $p=0.001$ ), E2F7 (HR=1.53, 95\% CI:1.13-2.07, and $p=0.006$ ), E2F8 (HR=1.47, 95\% CI:1.091.99 , and $p=0.012$ ) members was associated with poorer OS (overall survival) in ccRCC patients. However, the expression level of E2F6 was not associated with the OS of these patients. As shown in Figure 6B, with regard to progression-free survivals, higher mRNA expression of E2F1 ( $\mathrm{HR}=1.61,95 \% \mathrm{CI}: 1.17-2.21$, and $p=0.003)$, E2F2 $(\mathrm{HR}=1.57,95 \% \mathrm{CI}: 1.15-2.16$, and $p=0.005), \mathrm{E} 2 \mathrm{~F} 4(\mathrm{HR}=1.60,95 \% \mathrm{CI}: 1.14-2.23$, and $p=0.006), \mathrm{E} 2 \mathrm{~F} 7(\mathrm{HR}=2.23$, $95 \% \mathrm{CI}: 1.61-3.07$, and $p<0.001$ ), E2F8 (HR=1.87, 95\% CI:1.37-2.55, and $p<0.001$ ) members was associated with shorter progression-free survivals, while the expression of E2F3, E2F5, and E2F6 had no effect on the PFI of ccRCC patients. These results implied that mRNA expressions of E2F1/2/4/7/8 were significantly correlated with ccRCC patients' prognosis and they may act as useful biomarkers for prediction of clinical outcome including survival time and progression-free survival time.

\section{Independent Prognostic Value of mRNA Expression Levels of E2Fs in Terms of OS in ccRCC Patients}

Eight and three patients lacked of the data regarding the histologic grade and pathologic stage, respectively. After we found that there was significant association between mRNA expression levels of E2F1/2/3/4/5/7/8 and the OS in ccRCC patients, then the independent prognostic value of mRNA expression of E2Fs family was assessed in terms of OS for patients with ccRCC based on the TCGA database and prognostic data for Cox survival regression analysis. ${ }^{30}$ In the univariate analysis, we found that high mRNA expressions of E2F1 ( $\mathrm{HR}=1.594,95 \% \mathrm{CI}: 1.237-2.054$, and $p<0.001$ ), $\mathrm{E} 2 \mathrm{~F} 2(\mathrm{HR}=1.228,95 \% \mathrm{CI}: 0.734-2.057$, and $p<0.001)$, E2F3 ( $\mathrm{HR}=2.284,95 \% \mathrm{CI}: 1.495-3.489$, and $p<0.001)$, $\mathrm{E} 2 \mathrm{~F} 4(\mathrm{HR}=3.141,95 \% \mathrm{CI}: 1.929-5.115$, and $p<0.001)$, E2F5 (HR $=2.277,95 \% \mathrm{CI}: 1.536-3.376$, and $p<0.001)$, $\mathrm{E} 2 \mathrm{~F} 7(\mathrm{HR}=2.828,95 \% \mathrm{CI}:(2.148-3.725)$, and $p<0.001)$, and $\mathrm{E} 2 \mathrm{~F} 8(\mathrm{HR}=1.442,95 \% \mathrm{CI}:(1.049-1.981)$, and $p=$ 0.024 ) were associated with the poorer OS of ccRCC patients (Supplementary Table S1). Multivariate analysis for overall survival (OS) revealed that high mRNA expressions of E2F2 ( $\mathrm{HR}=0.310,95 \% \mathrm{CI}: 0.134-0.717$, and $p=0.006$ ), E2F4 $(\mathrm{HR}=2.837,95 \% \mathrm{CI}: 1.694-4.753$, and $p<0.001)$, E2F5 (HR $=2.364,95 \% \mathrm{CI}: 1.555-3.593$, and $p<0.001)$ and E2F7 $(\mathrm{HR}=2.094,95 \% \mathrm{CI}: 1.503-2.919$, and $p<0.001)$ were independently associated with shorter OS of patients with ccRCC. Finally, the results suggested that transcriptional expressions of E2F4/5/7 play independent prognostic roles in kidney cancer. 
A

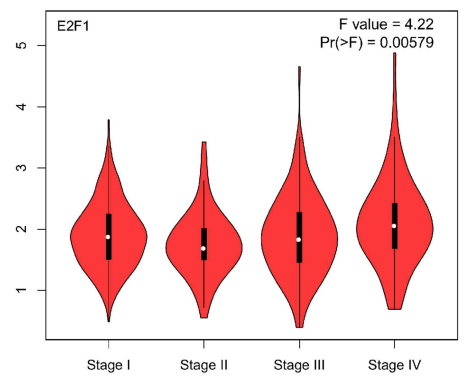

C

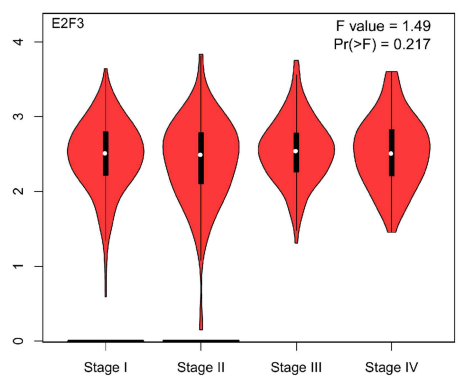

E

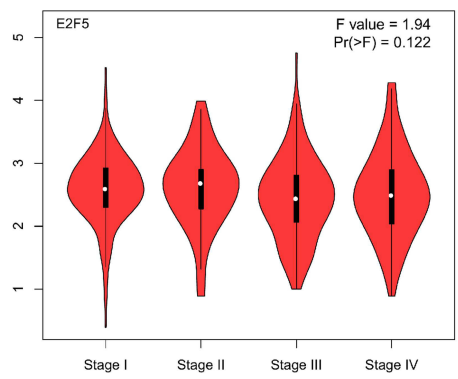

G

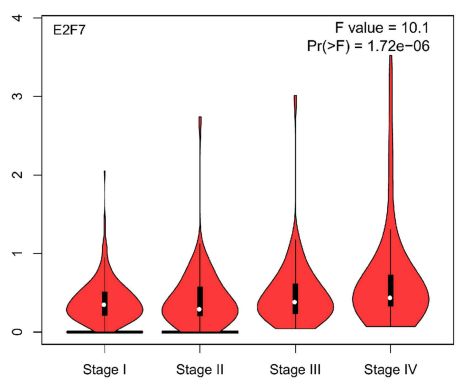

B

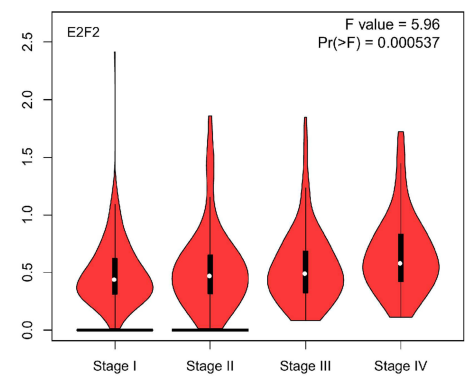

D

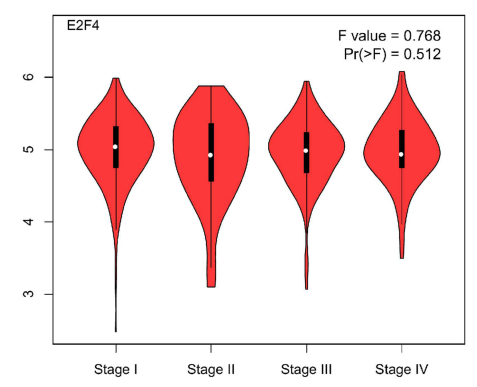

F

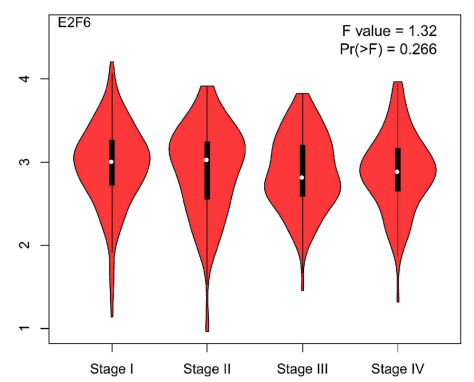

H

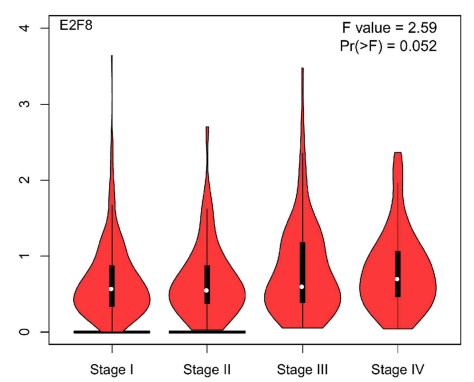

Figure 3 Correlations between E2Fs' mRNA level and clinical stages in ccRCC patients by GEPIA database were described with violin plots. The mRNA expressions of E2FI/2/7 were significantly related to patients' pathological stages $(\mathbf{A}-\mathbf{G})$, while mRNA expressions of E2F3/4/5/6/8 were not associated with patients' pathological stages (C-H). 
A

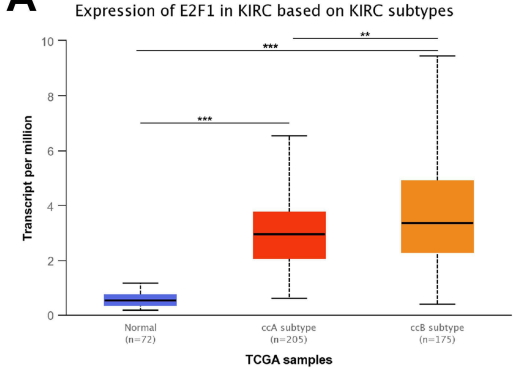

C

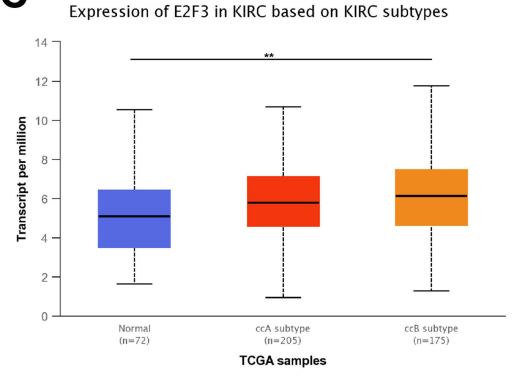

E

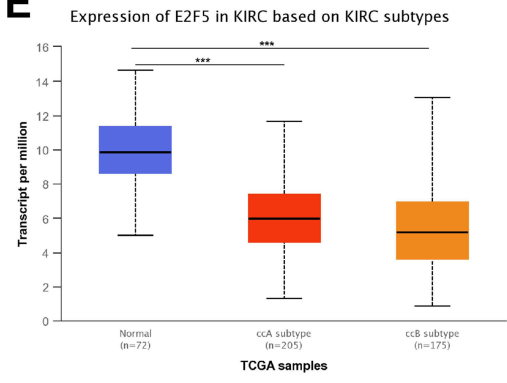

G

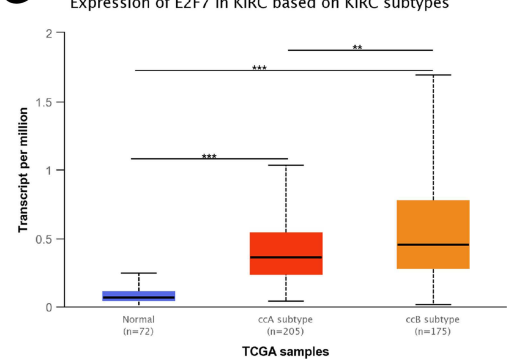

B

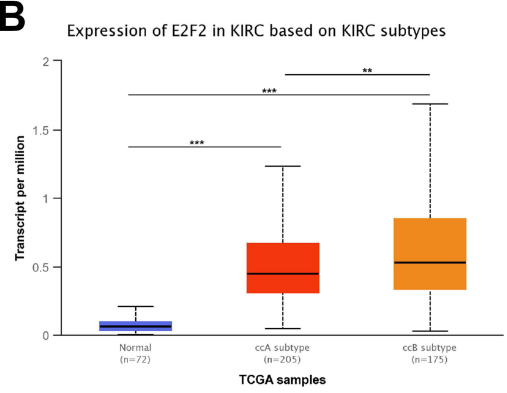

D

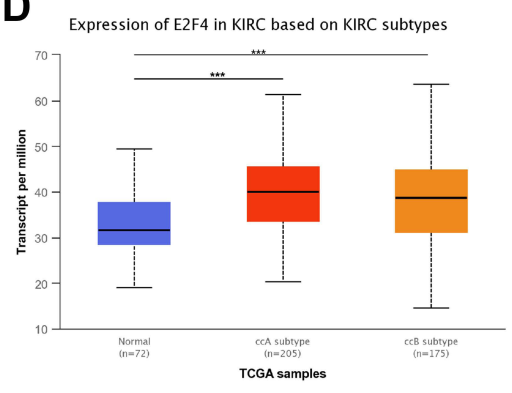

F Expression of E2F6 in KRCC based on KIRC subtryes

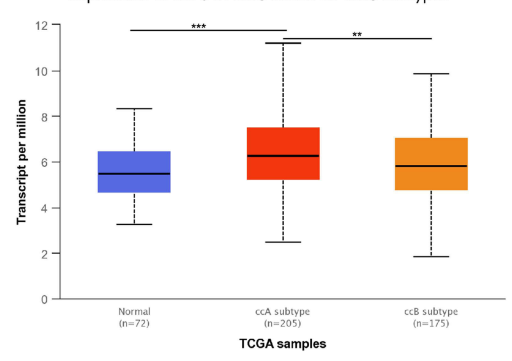

H Expression of E2F8 in KRC based on KIRC subtypes

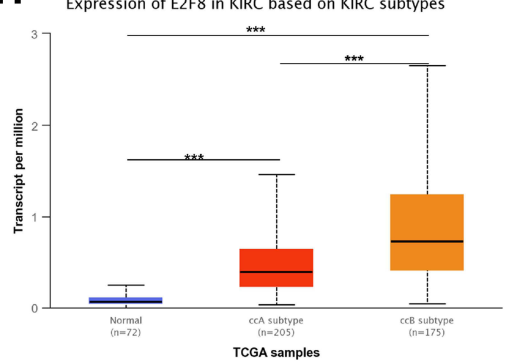

Figure 4 The mRNA levels of E2FI/2/3/4/5/6/7/8 in ccA and ccB subtypes of KIRC $(\mathbf{A}-\mathbf{H})$. ns indicates not significant; $* * p<0.01$; $* * p<00.00 \mathrm{I}$.

\section{Genetic Mutations Status in E2Fs and Their Associations with OS and Disease-Specific Survival (DSS) of ccRCC Patients}

To explore the potential expression pattern of E2Fs, we analyzed genetic alterations in E2Fs and their associations with OS and DSS of ccRCC patients by using the cBioPortal online tool. The genetic alteration of E2Fs in ccRCC is presented in Figure 7A, and the alteration frequency of E2Fs based on the cBioProtal database is illustrated in Figure 7B. In the 512 
A

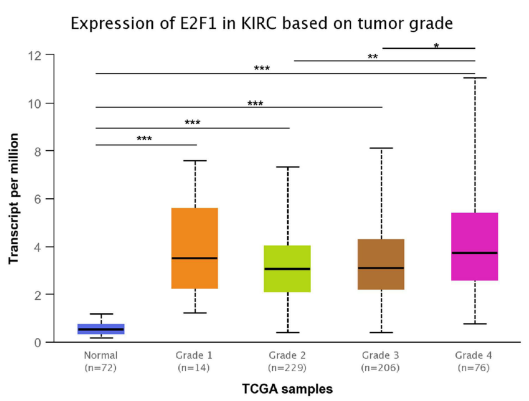

C

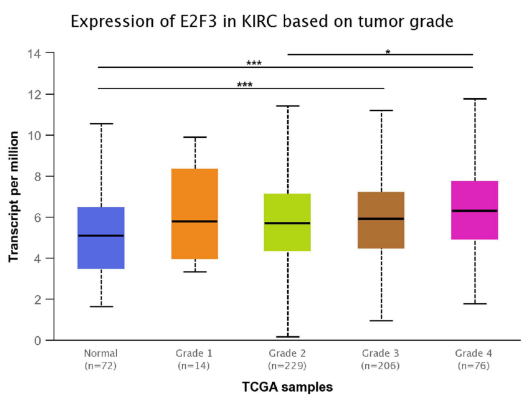

E

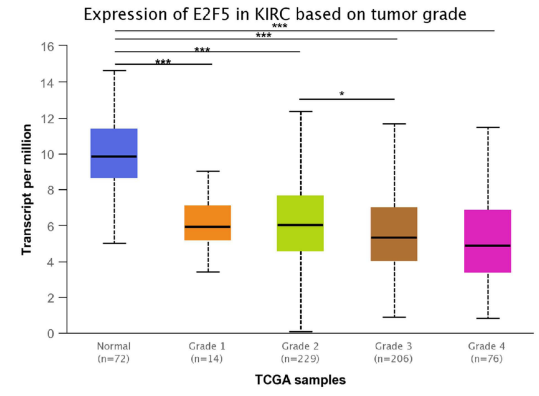

G

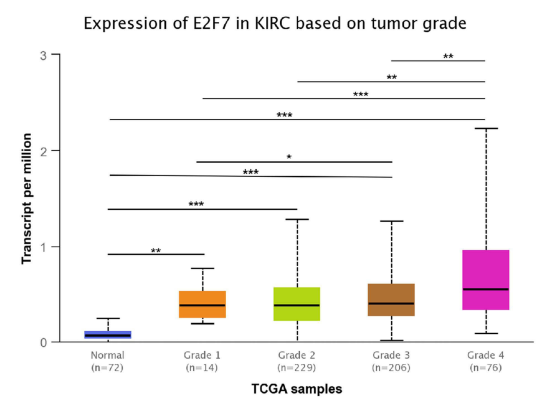

B

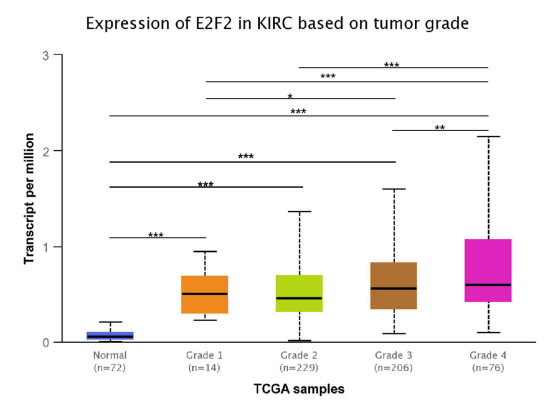

D

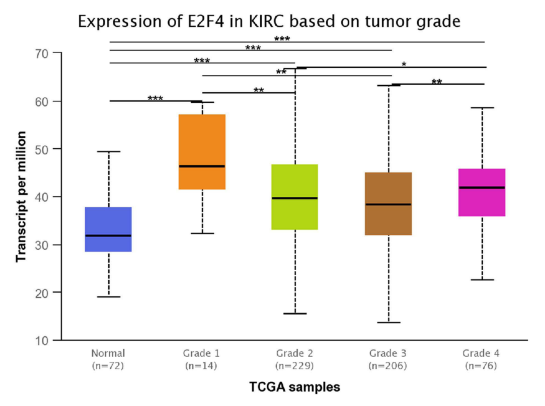

F

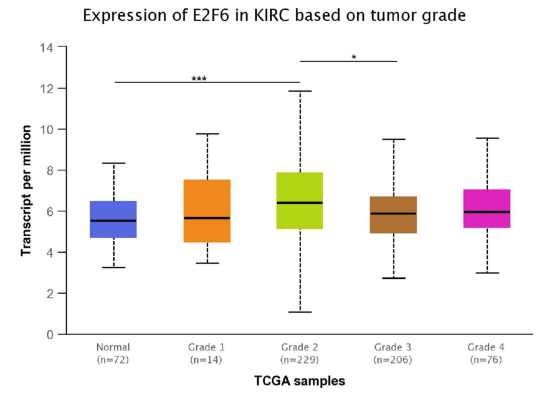

H

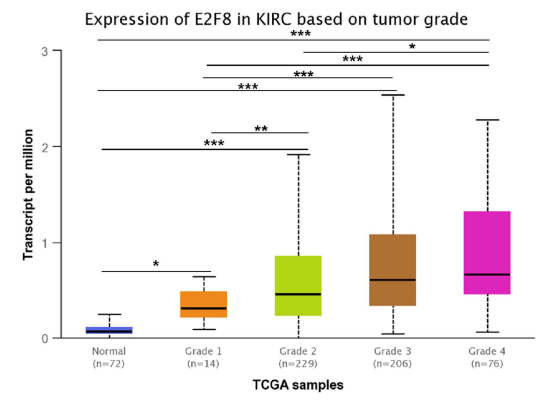

Figure 5 Associations of E2FI/2/3/4/5/6/7/8 expressions with tumor grades of $c c R C C$ were presented in $(\mathbf{A}-\mathbf{H})$, respectively. ns indicates not significant; ${ }^{*} p<0.05$; $* * p<$ $0.01 ; * * * p<0.001$. 
A
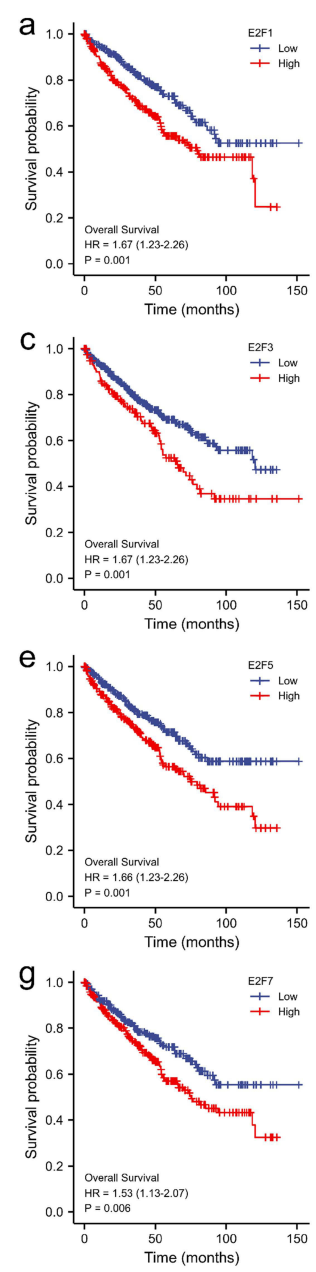

B
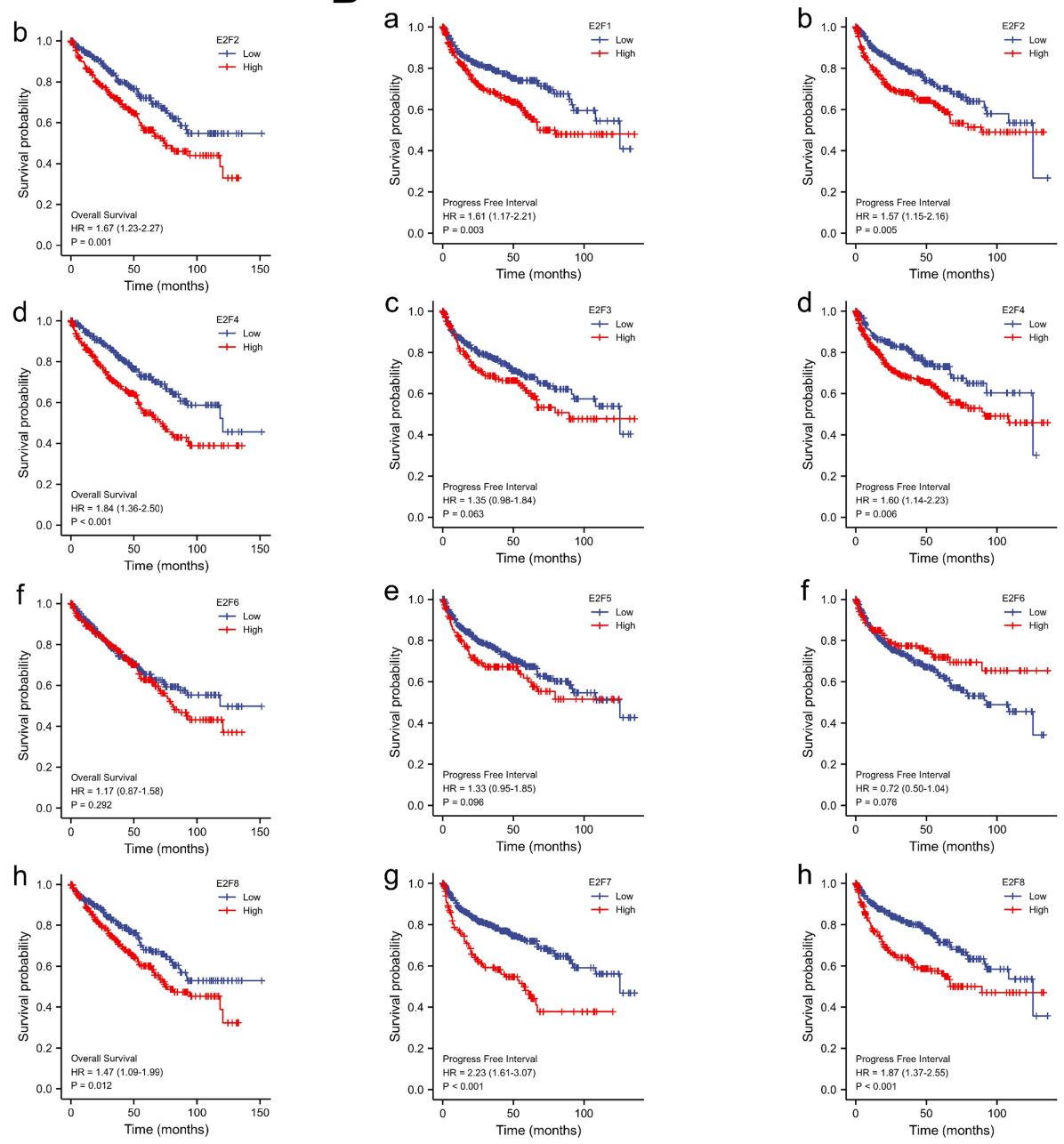

Figure 6 The overall survivals and progression-free survivals of ccRCC patients with high or low E2FI/2/3/4/5/6/7/8 expression were presented in (A and B), respectively.

sequenced ccRCC patients, genetic alteration was observed in 121 ccRCC patients, and the mutation rate was $24 \%$. The mutation rate of E2F1 was the highest, at a percentage of $7 \%$, compared with other E2Fs proteins. The mutation rate of $\mathrm{E} 2 \mathrm{~F} 2 / 3 / 4 / 7$ was $5 \%$, and the mutation rate of E2F5/8 was 4\%. Furthermore, the results were plotted using Kaplan-Meier plot and analyzed by Log rank test, which showed that genetic alteration in E2Fs was related to shorter OS (Figure 7C, $p=7.009 \mathrm{e}-3$ ) and PFS (Figure 7D, $p=5.389 \mathrm{e}-3$ ) of ccRCC patients. These results revealed that genetic alterations of E2Fs could also notably affect the prognosis of patients with ccRCC. The network between E2Fs and miRNAs are presented in Figure 7E.

\section{Predicted Functions and Pathways of the Changes in E2Fs and the 50 Most Frequently Altered Neighbor Genes in Patients with ccRCC}

After analyzing the E2Fs genetic alterations and their prognostic values in ccRCC patients, the 50 neighbor genes related to the E2Fs mutants were analyzed, and an integrated network was constructed based on the STRING database (https:// string-db.org/). As shown in Figure 8A, the cell cycle-related genes including CCNB2, CCNA2, FANCD2, CDC7, ORC6 and RAD54L were significantly associated with E2Fs mutations.

The functions of E2Fs and the 50 neighbor genes significantly associated with E2Fs were analyzed by GO (gene ontology) and KEGG (Kyoto Encyclopedia of Genes and Genomes) functional enrichment analyses using the ggplot2 $\mathrm{R}$ software package (Supplementary Table S2). As presented in Figure 8B, BP (biological processes) such as GO: 


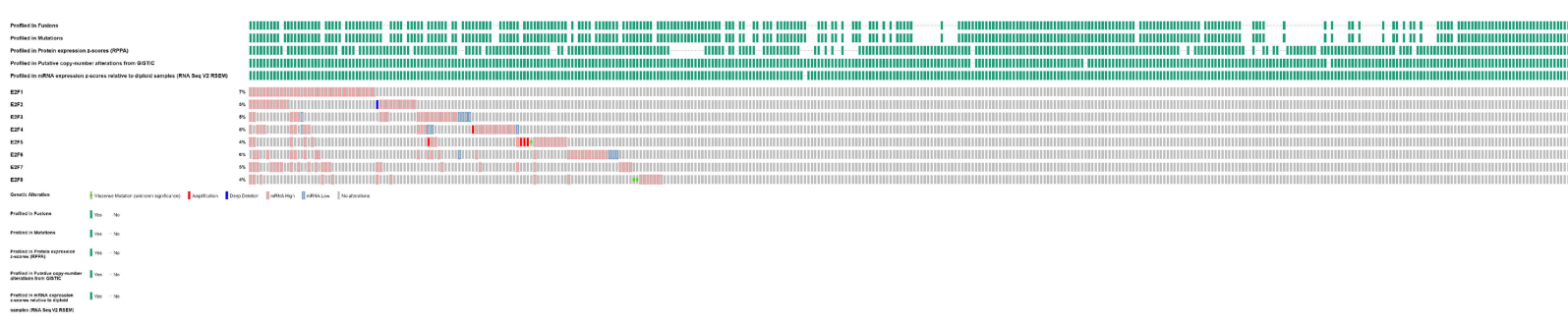

B

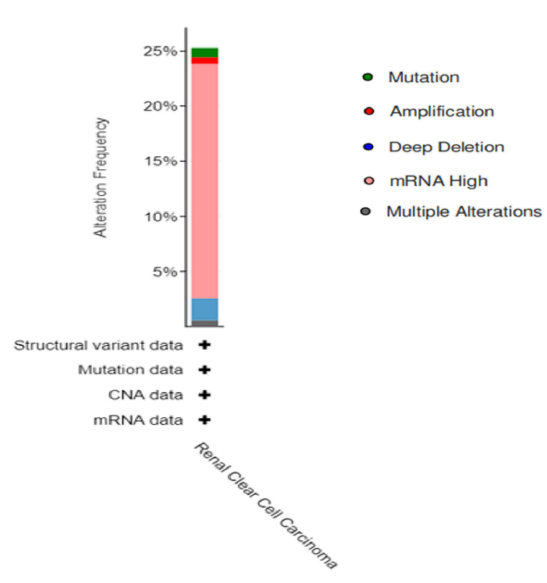

D

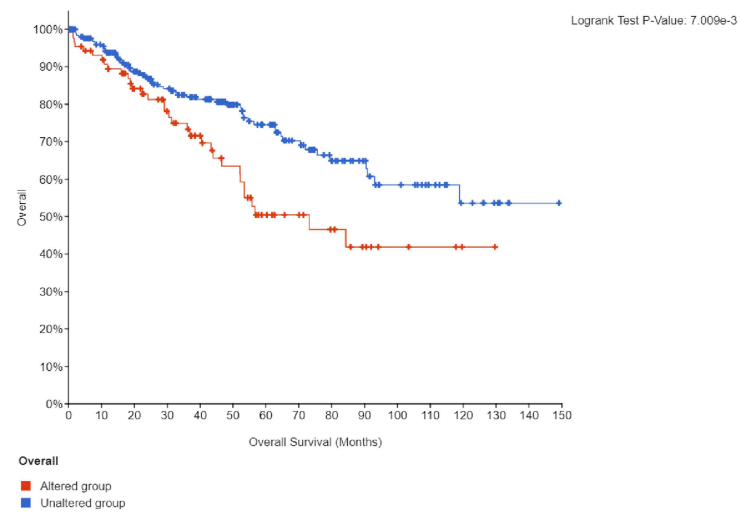

C

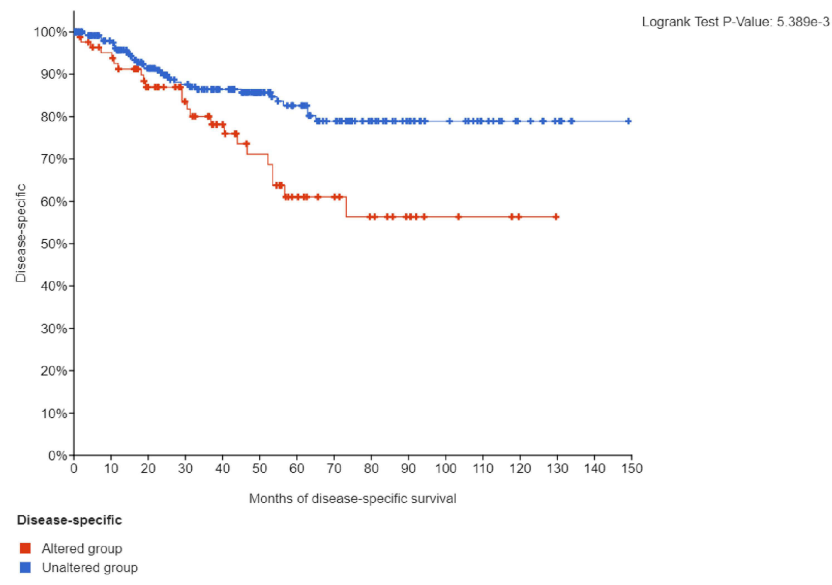

$\mathbf{E}$

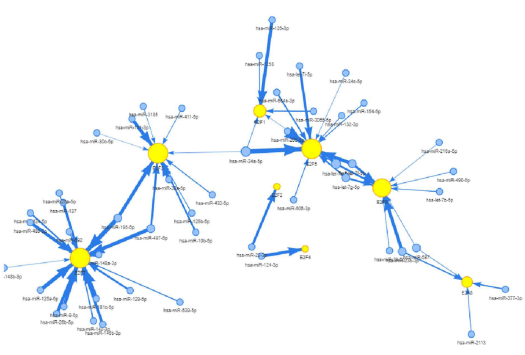

Figure 7 The genetic alteration of E2Fs in $\operatorname{ccRCC}(\mathbf{A})$ Alteration frequency of E2Fs based on the cBioProtal database (B) Kaplan-Meier plots revealed the overall survivals and progression-free survivals of ccRCC patients with or without E2Fs alterations (C and D). The network among E2Fs and miRNAs (E).

0006260 (DNA replication), GO: 0007093 (mitotic cell cycle checkpoint), GO:1901987 (regulation of cell cycle-phase transition) GO:0044843 (cell cycle G1/S phase transition) and GO: 0045930 (negative regulation of mitotic cell cycle) were significantly modulated by the E2Fs mutations in ccRCC. CC (cellular components), including GO: 0090575 (RNA polymerase II transcription factor complex), GO:0044798 (nuclear transcription factor complex), GO:0098687 
A

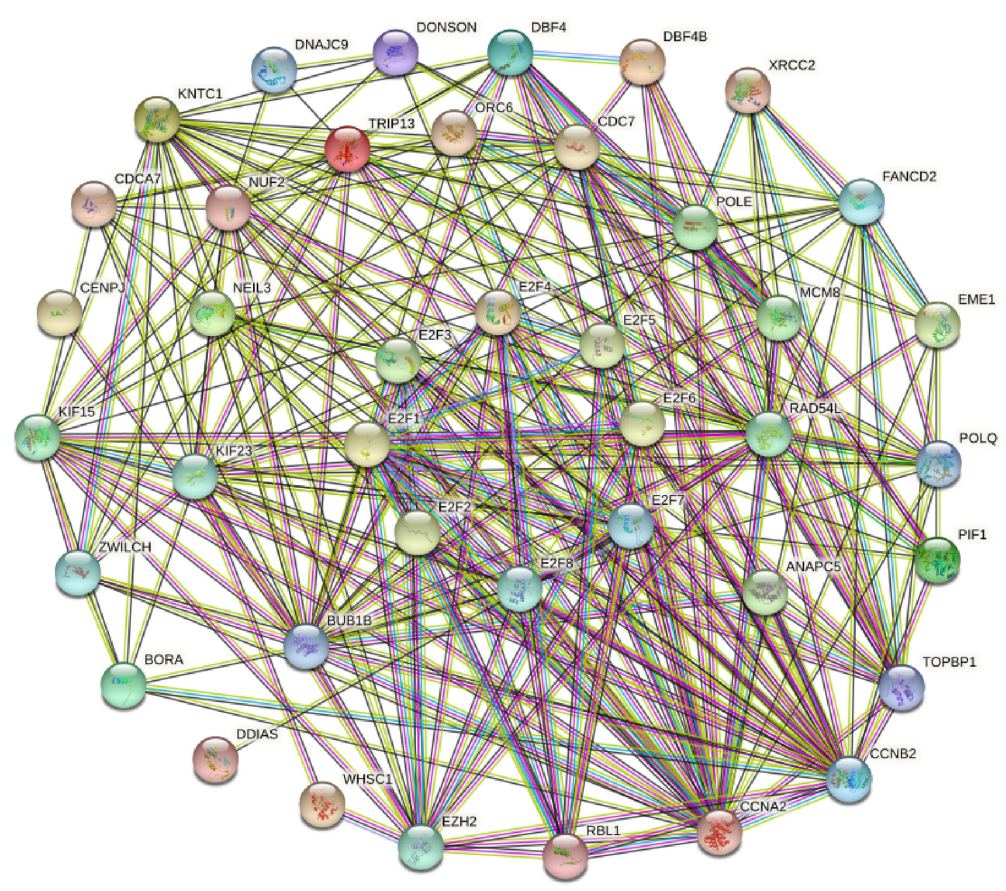

B

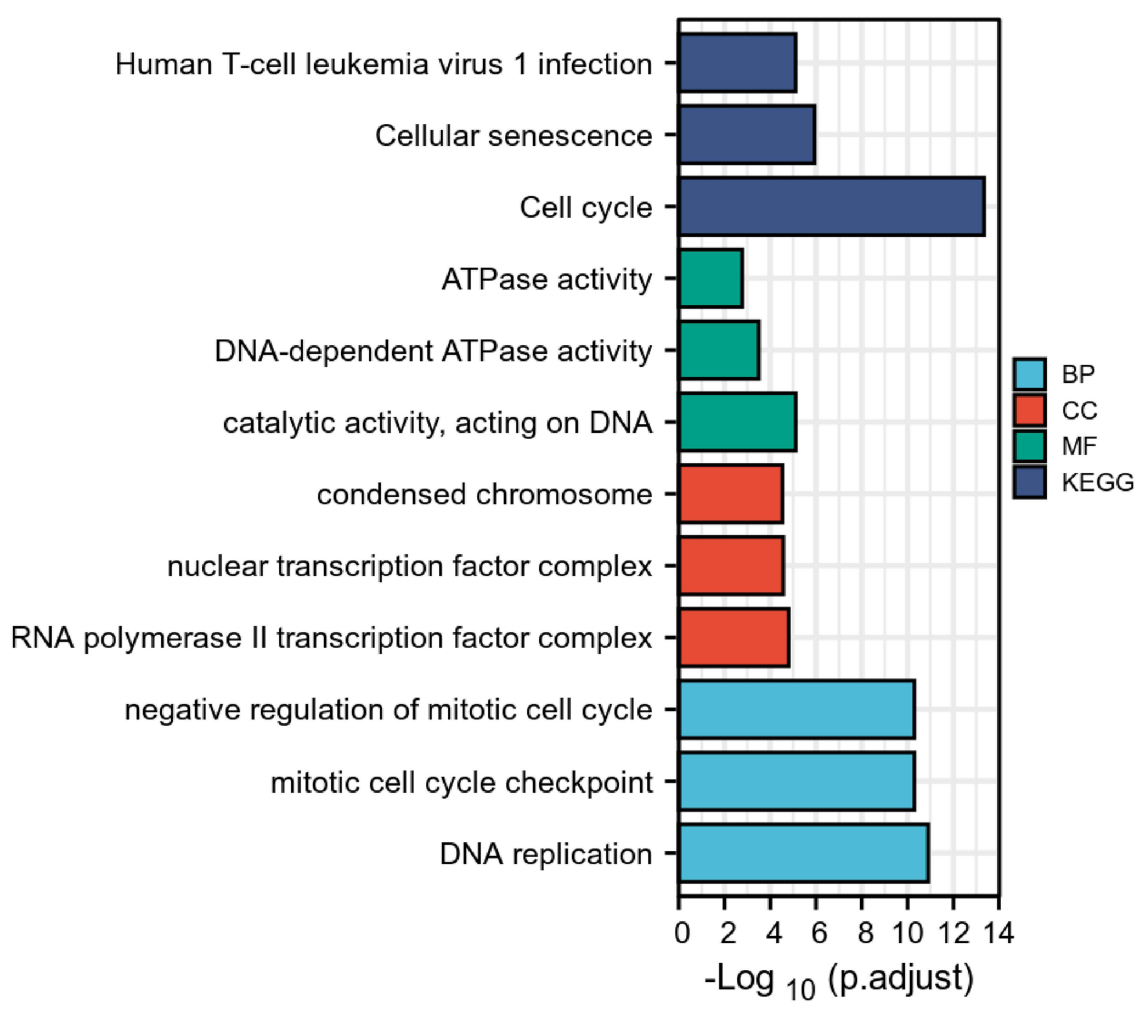

Figure 8 A network (A) and enrichment analysis (B) of E2Fs with the 50 neighboring genes related to the mutations of E2Fs in ccRCC.

(chromosomal region), GO:0005667 (transcription factor complex) and GO:0000793 (condensed chromosome) were significantly related to the E2Fs alterations. Furthermore, E2Fs family mutations also prominently affected the MF (molecular functions), such as GO:0140097 (catalytic activity, acting on DNA), GO:1990841 (promoter-specific 
chromatin binding), GO:0008094 (DNA-dependent ATPase activity), GO:0004386 (helicase activity) and GO:0016887 (ATPase activity). The enrichment analysis of KEGG pathways included 3 KEGG pathways, such as hsa04110 (cell cycle), hsa03440 (homologous recombination), hsa05219 (bladder cancer), hsa04218 (cellular senescence) and hsa05166 (human T-cell leukemia virus 1 infection), which were related to the functions of E2Fs mutations in ccRCC.

\section{Immune Infiltrations Analysis of the E2Fs Family in ccRCC}

Correlations between genes and immune infiltrations were estimated using TIMER. The positive connections existed between the abundance of CD8 + cell and the expression of all E2Fs, except for E2F1 and E2F5. The abundance of CD4 + cell was related to the expression of E2Fs but not E2F5 in a positive fashion. In addition, the abundance of macrophage, neutrophil, B, and dendritic cell positively showed positive associations with all the members of E2Fs (Figure 9A).

A
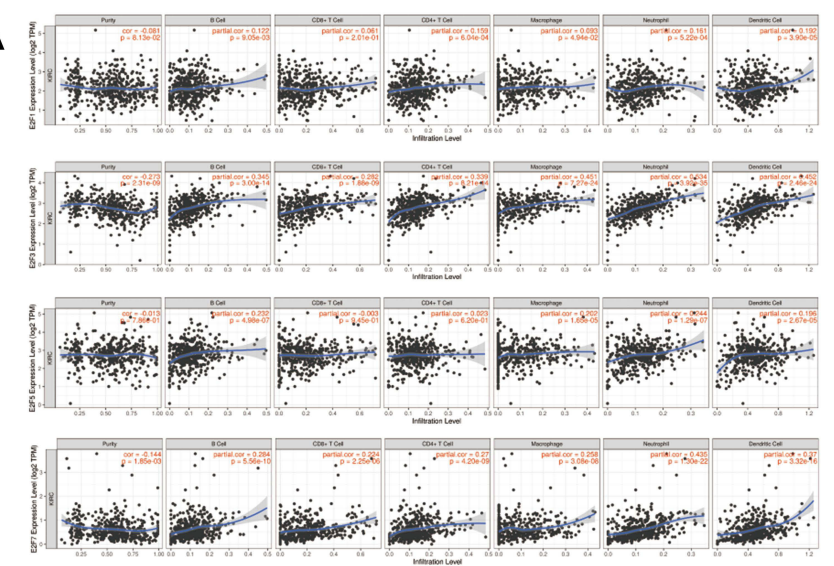

B
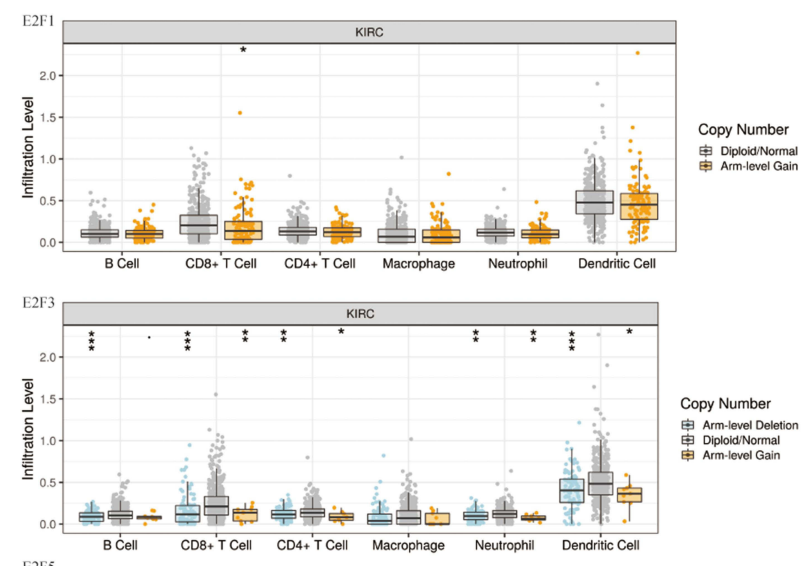

E2
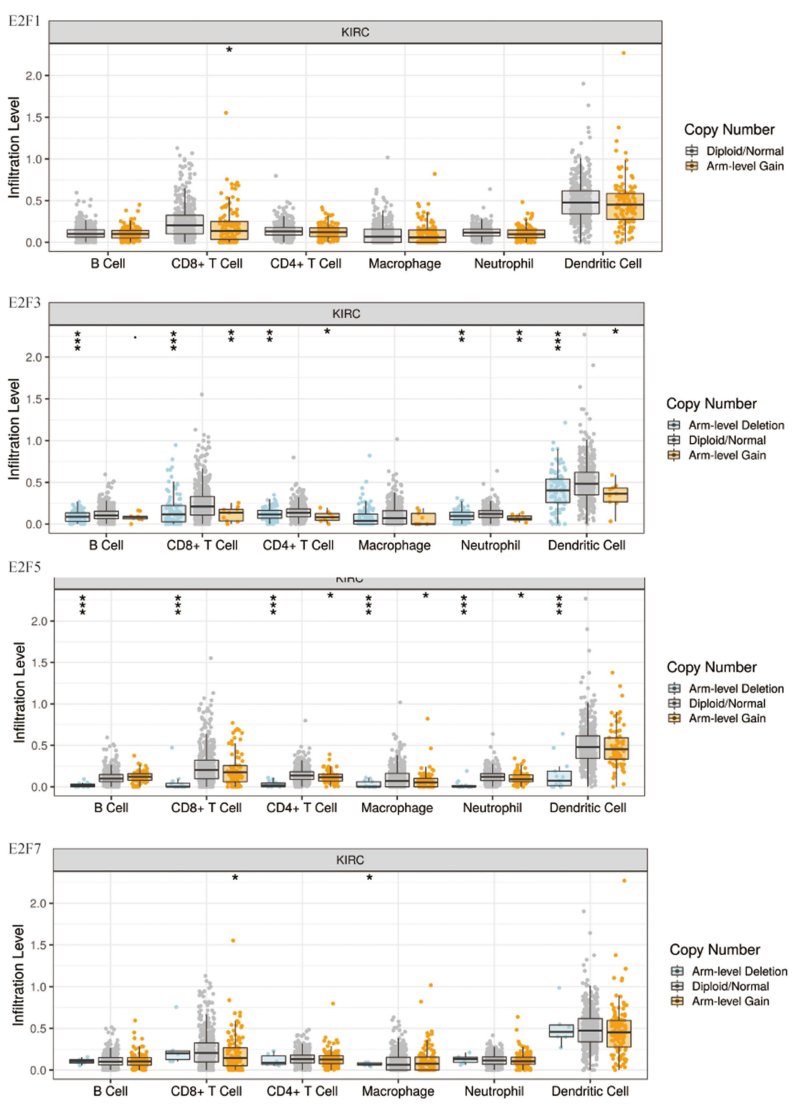
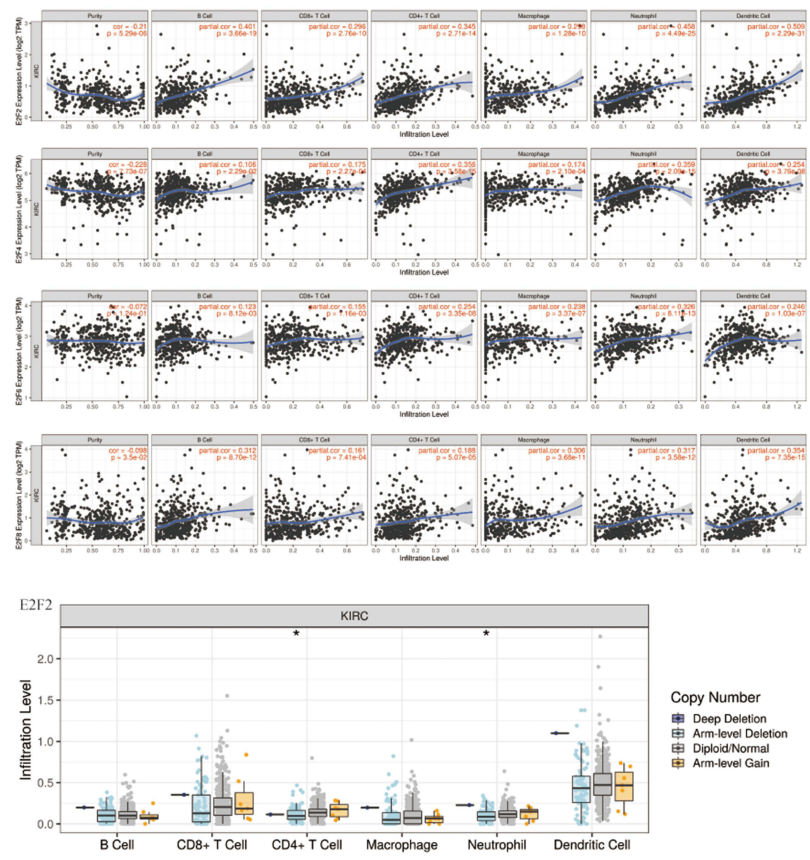

Copy Number

息 Deep Deletion

角 Diploiddovermal

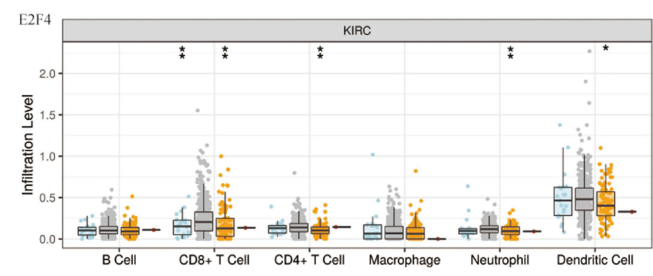

Copy Number

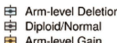

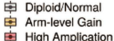

E2

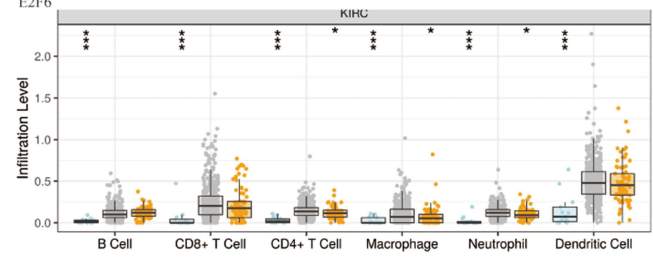

Copy Number

Arm-level Deletion
Diploidinormal

息 Diplodid Normal
Armlevel Gain

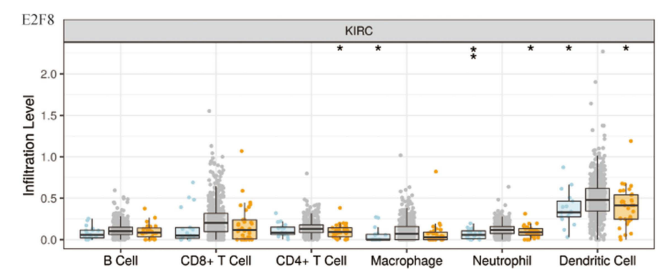

Copy Number

申 A Arm-level Deletion
帠 DiplodidNormal
Arm-level Gain

Figure 9 Association between E2Fs and crucial immune checkpoint genes $(\mathbf{A})$ and the comparison of tumor infiltration levels in ccRCC with different somatic copy number alterations for E2Fs (B) ns indicates not significant; $p \geq 0.05$; $p<<0.05$; $* *<0.01$; and $* * * p<0.001$. 
Furthermore, we turned nest to estimate the somatic copy number alterations (SCNA) of E2Fs. Results revealed that the SCNA of E2F8 significantly correlated with the infiltrating levels of CD4+ T cells, macrophages, neutrophils, and dendritic cells, while that of E2F4/6 was in significant connections with the infiltrating levels of B, CD8+ T, CD4+ T, neutrophils, and dendritic cells. However, only CD8+ T cells were significantly related to the SCNA of E2F1/5, while multiple immune cells, including B cells, CD8+ T cells, CD4+ T cells, neutrophils, and dendritic cells, were significantly relevant to the SCNA of E2F3. At the same time, the SCNA of E2F7 was significantly associated with macrophages and CD8+ T cells (Figure 9B).

Collectively, E2Fs families were closely related to the immune infiltration in patients bearing ccRCC.

\section{Verification of the Drug Sensitivity of the E2Fs Family}

For drug susceptibility analysis, through the GSCALite platform we analyzed the relationship between the E2Fs family expressions and multiple drug sensitivities (Figure 10). We found that the drug sensitivity of E2Fs was significantly associated with chemotherapy resistance and further confirm the E2Fs took part in multidrug resistance in ccRCC patients. It was well known that genetic mutations would lead to changes in drug sensitivity that affect clinical interventions, the results showed that the expression of E2F2/3/8 negatively correlated with drug resistance, indicating E2F2/3/8 can be used as new markers for drug sensitivity screening.

\section{Pathway Enrichment of E2Fs in ccRCC}

The pathways of the eight E2Fs were enriched with GSCALite. We found that 10 pathways were related to the function of the eight E2Fs in ccRCC (Figure 11). The expressions of E2Fs were in a high level in cell cycle and the expressions of E2F1/2/3/4/5/7/8 were also at a high level in apoptosis among 10 pathways. The expressions of E2Fs were almost inhibited in RAS/MAPK, RTK, and hormone estrogen receptor (ER) related pathway. These results indicated that changes in the target genes could influence multiple signaling pathways and the biological function.

\section{Discussion}

E2F transcription factors modulate extensive biological processes, whose activities are directly linked to cell cycle control, apoptosis, DNA damage response and transition. ${ }^{31}$ There is increasing evidence that E2Fs play an important role not only in tumorigenesis and proliferation of tumor cell, but also in tumor metastasis. However, the association between mRNA expression of distinct E2Fs family members and prognosis of ccRCC patients remained to be fully elucidated. In the current study, we systematically investigated the mRNA levels, genetic alteration, functional enrichment, immune infiltration, and prognostic value of the E2Fs family in patients with ccRCC.

E2F1 took part in a variety of cellular processes including cell cycle progression, DNA repair, DNA replication, cell differentiation, proliferation and apoptosis, which were highly correlated with the occurrence, development, metastasis and prognosis of tumors. Recent studies have shown that E2F1 is involved in metabolic reprogramming in tumor cells and plays an important role in tumor initiation and progression. ${ }^{32} \mathrm{E} 2 \mathrm{~F} 1$ could also act as a major transcriptional activator of metabolic genes in RCC. ${ }^{33}$ In our study, the expression of E2F1 was more highly expressed in ccRCC tissues than in normal tissues. Furthermore, we showed that high expression of E2F1 was significantly associated with shorter OS and PFI (progress free interval) in ccRCC patients, indicating E2F1 played a cancer-promoting role in renal clear cell carcinoma, while some research showed E2F1 was a tumor suppressor in prostate cancer. ${ }^{34}$

E2F2 was highly expressed in both gastric cancer tissues and cells compared with normal gastric tissues/cells, which promoted the migration and invasiveness of gastric cancer cells. ${ }^{35}$ It was also reported that the over-expressed miR-490$5 \mathrm{p}$ could inhibit the metastasis of hepatocellular carcinoma cells by down-regulating E2F2 expression levels. ${ }^{36}$ In our research, no significant differences were seen in the expression of E2F2 between normal tissues and carcinoma tissues in patients. The reason for this phenomenon may be that the E2F2 might be regulated by miR-22-3p according to the miRNA network (Figure 7E). A high E2F2 expression was significantly correlated with poor OS and PFI as well as individual cancer stages, suggesting an oncogenic role of E2F2.

Over-expressed E2F3 had been reported to take part in the development and progression of many cancers, including bladder cancer. ${ }^{37}$ MiR-22 suppressed the development and metastasis of bladder cancer cells by regulating E2F3. ${ }^{38}$ In our 

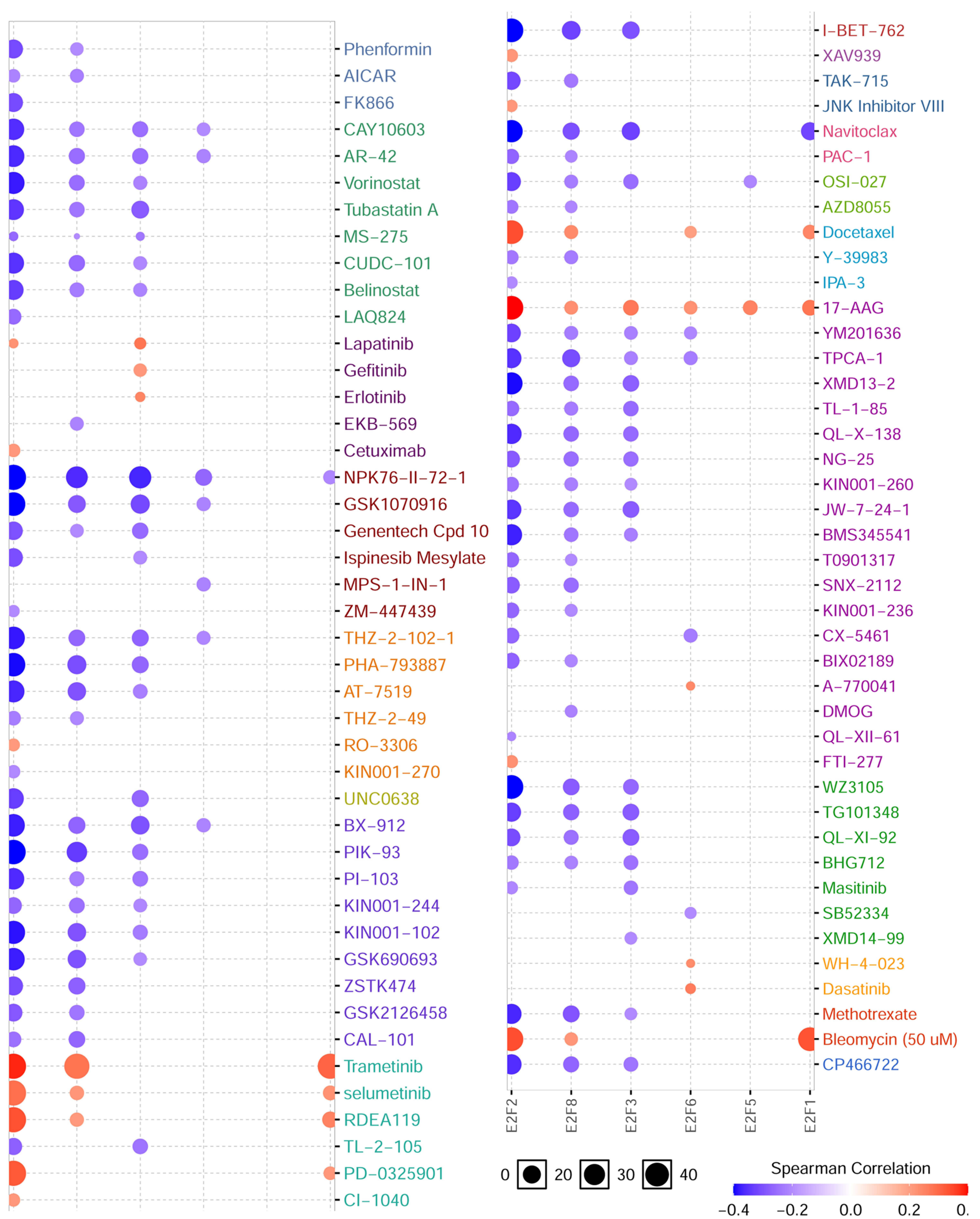

Figure 10 Drug resistance analysis of the E2Fs genes were completed using GSCALite. 


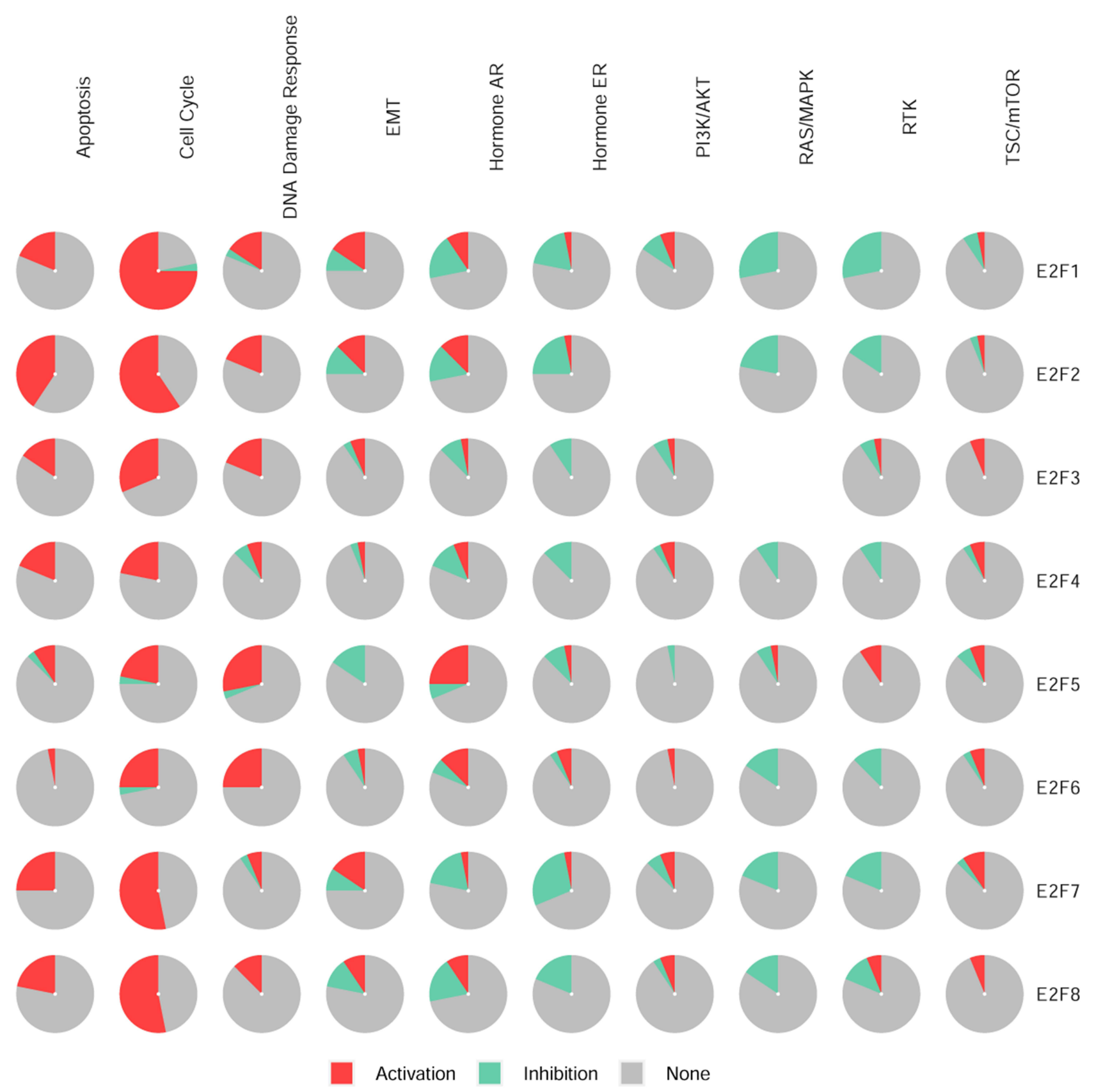

Figure II The pathway enrichment analysis of eight E2Fs genes (GSEALite).

study, we showed that the expression of E2F3 in ccRCC tissues was highly expressed in the cancer tissues relative to adjacent normal tissues, and this expression was not significantly associated with PFI and tumor stage except for OS in patients with ccRCC.

Amusingly, E2F4 could act as either a tumor suppressor in acute myeloid leukemia, ${ }^{39}$ or an oncogene in invasive breast carcinomas. ${ }^{40}$ In the current report, the expression of E2F4 was higher in kidney cancer tissues than in normal tissues, while this expression was not related to tumor stage. A high E2F4 expression was significantly associated with poor OS and PFI in ccRCC patients, which supported the role of E2F4 as a tumor oncogene. Besides, the expression of E2F4 played an independent prognostic role for OS in ccRCC.

E2F5 belonged to the E2F family of transcription factors and participated in cellular proliferation, apoptosis, differentiation and DNA damage response. E2F5 was aberrantly overexpressed in several tumors, such as breast 
tumor, ${ }^{41}$ and ovarian cancer. ${ }^{42}$ Knockdown of E2F5-induced cell death via the TP53-dependent pathway in breast cancer cells carrying wild-type TP53. ${ }^{41}$ E2F5, which was highly expressed in malignant tissues when compared to the nonmalignant tissues, promoted ovarian carcinogenesis by regulating Hippo and Wnt pathways. ${ }^{42}$ In this research, we showed that the expression of E2F5 in normal tissues was higher than that in kidney cancer tissues, but that there was no differential mRNA expression of E2F5 in ccRCC cell lines. According to the E2F5 expression in normal tissues compared with cancer tissues, it might play an inhibitory role, but a higher E2F5 expression showed a significant association with poor OS. Subsequently, we found that E2F5 was regulated by multiple non-coding RNAs, especially has-let-7f-5p, hsa-miR-205-5p and hsa-miR34a-5p, which might play an important role in promoting cancer. Besides, the expression of E2F5 played an independent prognostic role for OS in ccRCC.

E2F6, which was detected in several tumors, was highly expressed, such as ovarian cancer ${ }^{43}$ and gastric carcinoma. ${ }^{44}$ In this report, evidence from GEPIA and UALCAN databases showed that the expression of E2F6 were significantly elevated in clear cell renal cell cancer tissues compared to matched normal tissues, but this expression was not associated with tumor stage in patients. The E2F6 expression was not associated with poor OS or PFI. Furthermore, our study showed no difference in the levels of E2F6 between ccRCC and normal tissues.

Increased expression of E2F7 was found in glioblastoma patients, and high E2F7 expression showed a significant association with poor overall survival in glioblastoma patients. ${ }^{45}$ E2F7 might inhibit apoptosis and promote cell cycle of prostate cancer cells, which was regulated by miR-30c. ${ }^{46}$ In the study, the E2F7 expression was remarkably associated with patients' individual cancer stages. Correspondingly, the higher mRNA expression of E2F7 was significantly associated with shorter OS and PFI of ccRCC. In the multivariate analysis, the high E2F7 expression was an independent prognostic factor for the shorter OS of ccRCC.

E2F8 was a key transcription factor involved in many kinds of biological processes. MiR-223-5p suppressed tumor growth and metastasis in non-small cell lung cancer by targeting E2F8. ${ }^{47}$ Increased expression of E2F8 had been found in cervical cancer and was highly associated with the progression-free survival of cervical cancer patients. ${ }^{48}$ E2F8 in ER+ breast cancer cells conferred cisplatin resistance. ${ }^{49}$ In the present study, E2F8 was significantly overexpressed in ccRCC and E2F8 expression levels were correlated with the tumor stage of the ccRCC patients. Higher mRNA expression of E2F8 was also significantly related to shorter OS in ccRCC patients.

In this research, we comprehensively analyzed the expression and prognostic value of E2Fs in ccRCC cancer. Further, experimental demonstration was carried out to detect the E2Fs expressions in kidney cancer cell lines and ccRCC tissues. There were some limitations. First, although we exhibited that higher mRNA expressions of E2F4/5/7 were independent prognostic factors for shorter OS of patients with ccRCC, further investigation based on a large number of samples was needed to validate our findings and to explore the clinical application of the E2Fs members in the treatment of ccRCC. Second, we did not explore the potential mechanisms of distinct E2Fs. Further investigations were warranted to explore the potential mechanisms between E2Fs and ccRCC.

\section{Conclusions}

In the present study, E2F4/7 were highly expressed in ccRCC and significantly associated with worse pathological characteristics of ccRCC, including high pathological stage, poor molecular subtypes, high tumor grade, tumor immune cell infiltration, and drug sensitivity, consequently translating into poor OSs and PFSs of patients with ccRCC. Our results indicated that E2F4/7 could be potential biomarkers and therapeutic targets for ccRCC patients.

\section{Data Sharing Statement}

The original contributions presented in the study are included in the article.

\section{Ethics Approval and Consent to Participate}

This study was conducted in accordance with the Ethical Standards of the Institutional Ethics Committee of First Affiliated Hospital of Nanchang University and with the 1964 Helsinki declaration and its later amendments or comparable ethical standards. All patients provided informed consent. 


\section{Acknowledgments}

We thanked the two independent pathologists who confirmed the pathologic diagnosis of tumor tissues. Co-first authors: Chen Ru, Hu Bing, Zhang Zhicheng, and Jiang Ming. Co-corresponding authors: Fu Bin and Sun Ting.

\section{Funding}

There is no funding to report.

\section{Disclosure}

The authors declare that they have no conflicts of interest in this work.

\section{References}

1. Muller H, Bracken AP, Vernell R, et al. E2Fs regulate the expression of genes involved in differentiation, development, proliferation, and apoptosis. Genes Dev. 2001;15(3):267-285. doi:10.1101/gad.864201

2. Lin Z, Ren N, Jiang Y, Xu W, Shi Y, Liu G. Adenovirus-mediated E2F-1 gene transfer augments gemcitabine-induced apoptosis in human colon cancer cells. Clin Lab. 2015;61(10):1435-1444. doi:10.7754/clin.lab.2015.150104

3. Sozzani R, Maggio C, Varotto S, et al. Interplay between Arabidopsis activating factors E2Fb and E2Fa in cell cycle progression and development. Plant Physiol. 2006;140(4):1355-1366. doi:10.1104/pp.106.077990

4. Sun CC, Zhou Q, Hu W, et al. Transcriptional E2F1/2/5/8 as potential targets and transcriptional E2F3/6/7 as new biomarkers for the prognosis of human lung carcinoma. Aging. 2018;10(5):973-987. doi:10.18632/aging.101441

5. Manicum T, Ni F, Ye Y, Fan X, Chen BC. Prognostic values of E2F mRNA expression in human gastric cancer. Biosci Rep. 2018;38(6). doi:10.1042/BSR20181264

6. Santos M, Martinez-Fernandez M, Duenas M, et al. In vivo disruption of an Rb-E2F-Ezh2 signaling loop causes bladder cancer. Cancer Res. 2014;74(22):6565-6577. doi:10.1158/0008-5472.CAN-14-1218

7. Shackney SE, Chowdhury SA, Schwartz R, Novel A. Subset of human tumors that simultaneously overexpress multiple E2F-responsive genes found in breast, ovarian, and prostate cancers. Cancer Inform. 2014;13(Supp15):89-100. doi:10.4137/CIN.S14062

8. Rennhack J, Andrechek E. Conserved E2F mediated metastasis in mouse models of breast cancer and HER2 positive patients. Oncoscience. 2015;2 (10):867-871. doi:10.18632/oncoscience.259

9. Sung H, Ferlay J, Siegel RL, et al. Global cancer statistics 2020: GLOBOCAN estimates of incidence and mortality worldwide for 36 cancers in 185 countries. CA Cancer J Clin. 2021;71(3):209-249. doi:10.3322/caac.21660

10. Laguna MP. Re: international variations and trends in renal cell carcinoma incidence and mortality. $J$ Urol. 2015;194(4):950-951. doi:10.1016/j. juro.2015.07.002

11. Xu X, Wu J, Li S, et al. Downregulation of microRNA-182-5p contributes to renal cell carcinoma proliferation via activating the AKT/FOXO3a signaling pathway. Mol Cancer. 2014;13:109. doi:10.1186/1476-4598-13-109

12. Ljungberg B, Albiges L, Abu-Ghanem Y, et al. European association of urology guidelines on renal cell carcinoma: the 2019 update. Eur Urol. 2019;75(5):799-810. doi:10.1016/j.eururo.2019.02.011

13. Ljungberg B, Bensalah K, Canfield S, et al. EAU guidelines on renal cell carcinoma: 2014 update. Eur Urol. 2015;67(5):913-924. doi:10.1016/j. eururo.2015.01.005

14. Motzer RJ, Molina AM. Targeting renal cell carcinoma. J Clin Oncol. 2009;27(20):3274-3276. doi:10.1200/JCO.2009.21.8461

15. Cen J, Liang Y, Huang Y, et al. Circular RNA circSDHC serves as a sponge for miR-127-3p to promote the proliferation and metastasis of renal cell carcinoma via the CDKN3/E2F1 axis. Mol Cancer. 2021;20(1):19. doi:10.1186/s12943-021-01314-w

16. Ding M, Lu X, Wang C, et al. The E2F1-miR-520/372/373-SPOP axis modulates progression of renal carcinoma. Cancer Res. 2018;78 (24):6771-6784. doi:10.1158/0008-5472.CAN-18-1662

17. Gao Y, Li H, Ma X, et al. KLF6 suppresses metastasis of clear cell renal cell carcinoma via transcriptional repression of E2F1. Cancer Res. 2017;77 (2):330-342. doi:10.1158/0008-5472.CAN-16-0348

18. Ma X, Gao Y, Fan Y, et al. Overexpression of E2F1 promotes tumor malignancy and correlates with TNM stages in clear cell renal cell carcinoma. PLoS One. 2013;8(9):e73436. doi:10.1371/journal.pone.0073436

19. Guo R, Zou B, Liang Y, et al. LncRNA RCAT1 promotes tumor progression and metastasis via miR-214-5p/E2F2 axis in renal cell carcinoma. Cell Death Dis. 2021;12(7):689. doi:10.1038/s41419-021-03955-7

20. Gao Y, Ma X, Yao Y, et al. miR-155 regulates the proliferation and invasion of clear cell renal cell carcinoma cells by targeting E2F2. Oncotarget. 2016;7(15):20324-20337. doi:10.18632/oncotarget.7951

21. Qiu M, Liang Z, Chen L, et al. MicroRNA-200c suppresses cell growth and metastasis by targeting Bmi-1 and E2F3 in renal cancer cells. Exp Ther Med. 2017;13(4):1329-1336. doi:10.3892/etm.2017.4147

22. Kim YS, Jung J, Jeong H, et al. Protein expression profiles and prognostic value of E2F family members in clear cell renal cell carcinoma. Pathol Res Pract. 2020;216(4):152880. doi:10.1016/j.prp.2020.152880

23. Cai Q, Zhao A, Ren LG, et al. MiR-425 involves in the development and progression of renal cell carcinoma by inhibiting E2F6. Eur Rev Med Pharmacol Sci. 2018;22(19):6300-6307. doi:10.26355/eurrev_201810 16040

24. Cheng C, Guo L, Ma Y, Wang Z, Fan X, Shan Z. Up-regulation of miR-26a-5p inhibits E2F7 to regulate the progression of renal carcinoma cells. Cancer Manag Res. 2020;12:11723-11733. doi:10.2147/CMAR.S271710

25. Chandrashekar DS, Bashel B, Balasubramanya SAH, et al. UALCAN: a portal for facilitating tumor subgroup gene expression and survival analyses. Neoplasia. 2017;19(8):649-658. doi:10.1016/j.neo.2017.05.002 
26. Tang Z, Li C, Kang B, Gao G, Li C, Zhang Z. GEPIA: a web server for cancer and normal gene expression profiling and interactive analyses. Nucleic Acids Res. 2017;45(W1):W98-W102. doi:10.1093/nar/gkx247

27. Gao J, Aksoy BA, Dogrusoz U, et al. Integrative analysis of complex cancer genomics and clinical profiles using the cBioPortal. Sci Signal. 2013;6 (269):pl1. doi:10.1126/scisignal.2004088

28. Szklarczyk D, Gable AL, Lyon D, et al. STRING v11: protein-protein association networks with increased coverage, supporting functional discovery in genome-wide experimental datasets. Nucleic Acids Res. 2019;47(D1):D607-D613. doi:10.1093/nar/gky1131

29. Li B, Severson E, Pignon JC, et al. Comprehensive analyses of tumor immunity: implications for cancer immunotherapy. Genome Biol. 2016;17 (1):174. doi:10.1186/s13059-016-1028-7

30. Liu J, Lichtenberg T, Hoadley KA, et al. An integrated TCGA pan-cancer clinical data resource to drive high-quality survival outcome analytics. Cell. 2018;173(2):400-416 e11. doi:10.1016/j.cell.2018.02.052

31. Kent LN, Leone G. The broken cycle: E2F dysfunction in cancer. Nat Rev Cancer. 2019;19(6):326-338. doi:10.1038/s41568-019-0143-7

32. Wu M, Seto E, Zhang J. E2F1 enhances glycolysis through suppressing Sirt6 transcription in cancer cells. Oncotarget. $2015 ; 6(13): 11252-11263$. doi:10.18632/oncotarget.3594

33. Pandey N, Lanke V, Vinod PK. Network-based metabolic characterization of renal cell carcinoma. Sci Rep. 2020;10(1):5955. doi:10.1038/s41598020-62853-8

34. Chun JN, Cho M, Park S, So I, Jeon JH. The conflicting role of E2F1 in prostate cancer: a matter of cell context or interpretational flexibility? Biochim Biophys Acta Rev Cancer. 2020;1873(1):188336. doi:10.1016/j.bbcan.2019.188336

35. Li H, Zhao S, Shen L, et al. E2F2 inhibition induces autophagy via the PI3K/Akt/mTOR pathway in gastric cancer. Aging. 2021;13 (10):13626-13643. doi:10.18632/aging.202891

36. Fang ZQ, Li MC, Zhang YQ, Liu XG. MiR-490-5p inhibits the metastasis of hepatocellular carcinoma by down-regulating E2F2 and ECT2. J Cell Biochem. 2018;119(10):8317-8324. doi:10.1002/jcb.26876

37. Zhang Y, Zhang Z, Li Z, et al. MicroRNA-497 inhibits the proliferation, migration and invasion of human bladder transitional cell carcinoma cells by targeting E2F3. Oncol Rep. 2016;36(3):1293-1300. doi:10.3892/or.2016.4923

38. Guo J, Zhang J, Yang T, Zhang W, Liu M. MiR-22 suppresses the growth and metastasis of bladder cancer cells by targeting E2F3. Int J Clin Exp Pathol. 2020;13(3):587-596.

39. Feng Y, Li L, Du Y, Peng X, Chen F. E2F4 functions as a tumour suppressor in acute myeloid leukaemia via inhibition of the MAPK signalling pathway by binding to EZH2. J Cell Mol Med. 2020;24(3):2157-2168. doi:10.1111/jcmm.14853

40. Rakha EA, Pinder SE, Paish EC, Robertson JF, Ellis IO. Expression of E2F-4 in invasive breast carcinomas is associated with poor prognosis. J Pathol. 2004;203(3):754-761. doi:10.1002/path.1573

41. Inagaki Y, Wu D, Fujiwara K, et al. Knockdown of E2F5 induces cell death via the TP53-dependent pathway in breast cancer cells carrying wild-type TP53. Oncol Rep. 2020;44(5):2241-2252. doi:10.3892/or.2020.7761

42. Malgundkar SH, Burney I, Al Moundhri M, et al. E2F5 promotes the malignancy of ovarian cancer via the regulation of hippo and wnt pathways. Genet Test Mol Biomark. 2021;25(3):179-186. doi:10.1089/gtmb.2020.0166

43. An Y, Zhang J, Cheng X, et al. miR-454 suppresses the proliferation and invasion of ovarian cancer by targeting E2F6. Cancer Cell Int. 2020;20:237. doi:10.1186/s12935-020-01300-0

44. Li Y, Jiang L, Lv S, et al. E2F6-mediated lncRNA CASC2 down-regulation predicts poor prognosis and promotes progression in gastric carcinoma. Life Sci. 2019;232:116649. doi:10.1016/j.1fs.2019.116649

45. Yang R, Wang M, Zhang G, et al. E2F7-EZH2 axis regulates PTEN/AKT/mTOR signalling and glioblastoma progression. Br J Cancer. 2020;123 (9):1445-1455. doi:10.1038/s41416-020-01032-y

46. Wang Y, Pei X, Xu P, et al. E2F7, regulated by miR30c, inhibits apoptosis and promotes cell cycle of prostate cancer cells. Oncol Rep. 2020;44 (3):849-862. doi:10.3892/or.2020.7659

47. Dou L, Han K, Xiao M, Lv F. miR-223-5p suppresses tumor growth and metastasis in non-small cell lung cancer by targeting E2F8. Oncol Res. 2019;27(2):261-268. doi:10.3727/096504018X15219188894056

48. Kim LK, Park SA, Eoh KJ, Heo TH, Kim YT, Kim HJ. E2F8 regulates the proliferation and invasion through epithelial-mesenchymal transition in cervical cancer. Int J Biol Sci. 2020;16(2):320-329. doi:10.7150/ijbs.37686

49. Tian J, Lin Y, Yu J. E2F8 confers cisplatin resistance to ER+ breast cancer cells via transcriptionally activating MASTL. Biomed Pharmacother. 2017;92:919-926. doi:10.1016/j.biopha.2017.05.118

\section{Publish your work in this journal}

The International Journal of General Medicine is an international, peer-reviewed open-access journal that focuses on general and internal medicine, pathogenesis, epidemiology, diagnosis, monitoring and treatment protocols. The journal is characterized by the rapid reporting of reviews, original research and clinical studies across all disease areas. The manuscript management system is completely online and includes a very quick and fair peer-review system, which is all easy to use. Visit http://www.dovepress.com/testimonials.php to read real quotes from published authors.

Submit your manuscript here: https://www.dovepress.com/international-journal-of-general-medicine-journal 